\title{
Determinants and Trends of Under-Five Child Mortality in Ethiopia: A Multi-level Logistic Modeling Approach
}

\author{
Mulugeta Tadesse Ankea \\ Department of Statistics, Natural and Computational Science, Addis Ababa University, Addis Ababa, Ethiopia
}

Email address:

Muletade2@yahoo.com,mulertadesse19@gmail.com

To cite this article:

Mulugeta Tadesse Ankea. Determinants and trends of Under-Five Child Mortality in Ethiopia: A Multi-level Logistic Modeling Approach. International Journal of Biomedical Science and Engineering. Vol. 9, No. 3, 2021, pp. 37-58. doi: 10.11648/j.ijbse.20210903.11

Received: June 26, 2021; Accepted: July 23, 2021; Published: August 6, 2021

\begin{abstract}
The burden of under-five mortality remains unevenly distributed. About 80 percent of under-five deaths occur in two regions, sub-Saharan Africa and South Asia. Ethiopia is among the six countries that account for half of the global under-five deaths. The aim of this study was to identify the significant socio-economic and demographic factors influencing under-five child mortality and evaluate the variation among the regional states of Ethiopia. In this study, the 2000, 2005, 2011 and 2016 EDHS data were used to describe the trend of under-five mortality in Ethiopia. The 2016 EDHS data have been used to analyze determinants and variation of under-five mortality by background characteristics. Single-level logistic regression and multilevel logistic regression models were used to identify the major risk factors of under-five mortality and regional variations in under-five child mortality in Ethiopia using the 2016 EDHS data. The results from single-level and multilevel logistic regression analyses showed that Sex of a child, Age of a child in month, Birth type, Birth order number, Number of Household size, Breastfeeding status, Educational level of mother's, Place of residence and type of toilet facility had significant effects on under-five child mortality and there is variation of under-five child mortality from region to region. Conversely, preceding birth interval, wealth index Household, Source of drinking water and place of delivery were found insignificant. The results revealed variation of under-five child mortality from region to region. The multilevel logistic regression analysis result showed that the effects of breastfeeding varied across regions whereas the effects of other covariates on under-five child mortality remained fixed across regions.
\end{abstract}

Keywords: EDHS 2016 Data, Ethiopia, Multilevel Level Logistic Model, Under-five Child Mortality

\section{Introduction}

\subsection{Background of the Study}

One of the demographic variables that affect population trends/growth is mortality. Infant and child mortality rates are used as summary indicators of social development, quality of life, overall health, child health, maternal health and welfare. Under-five child mortality is a leading indicator of child health and overall development of a nation [5]. Child mortality rates also reflect a country's level of the social-economic development and quality of life and are used for monitoring and evaluating population, health programs and policies. Child mortality rates are unacceptably high in many developing countries and need to remain the focus of public policy to gain improvement in infant and child survival.
Every year, millions of children under 5 years of age die, largely from preventable causes [32]. In almost half of the cases, malnutrition plays a role, while unsafe water, inadequate sanitation and hygiene are also significant contributing factors. The world has made substantial progress in reducing child mortality in the past few decades. Estimates Developed by the UN Inter-agency Group for Child Mortality Estimation [32] shows that the global under- five mortality rate declined by 56 percent from 93 deaths per 1,000 live births in 1990 to 41 in 2016. Progress in reducing child mortality has been accelerated during the 2000-2016 period compared with the 1990s. That is, the annual rate of reduction in the under- five mortality rate has increased from 1.9 percent during $1990-2000$ to 4.0 percent during 2000 2016. In 2016, an estimated 5.6 million children died before their fifth birthday of which 2.6 million (46 percent) died in the first month of life. It is unacceptable that 15,000 children 
die every day, mostly from preventable causes and treatable diseases, even though the knowledge and technologies for life-saving interventions are available.

At country level, the under-five mortality rate ranged from a high of 133 deaths per 1,000 live births to a low of 2 deaths per 1,000 live births in 2016. Many countries still have very high rates - about 80 percent of under- five deaths occur in two regions, sub-Saharan Africa and Southern Asia, Six countries account for half of the global under- five deaths, namely, India, Nigeria, Pakistan, the Democratic Republic of the Congo, Ethiopia and China.

Sub-Saharan Africa remains the region with the highest under- five mortality rate in the world. In 2016, the region had an average under- five mortality rate of 79 deaths per 1,000 live births. This translates to 1 child in 13 dying before his or her fifth birthday - 15 times higher than the average ratio of 1 in 189 in high-income countries, or 20 times higher than the ratio of 1 in 250 in the region of Australia and New Zealand. The child health status isalways the focus of the health studies in developing countries. It reflects the healthy status and living level and the healthy status in the childhood finally reflects the healthy, income and achievement in the whole life. Children are at greater risk of dying before age five. Reducing child mortality rates has been one of the eight 'Millenniums Development Goals' of the United Nations. Worldwide, under-five child mortality is reducing. But in the developing countries, almost 11 million children are dying every year and two thirds of the deaths are preventable. The unsafe water, malnutrition, the lack of education, health care and social services are the major factors which slow down the reduction rate in some regions. Hence, analysis of child mortality and the methods to reduce the rate are imperative. The high rate countries centralize around Africa, South America and the Southeast Asia [36].

The 2016 EDHS reported that Ethiopia has successfully reduced the under-five child mortality rate by two-third between 2000 and 2015, which was the target for achieving Millennium Development Goal-4 [6]. However, the under-five mortality rate in Ethiopia is still higher than the under-five mortality rates of other several low and middle income countries (LMIC). According to the Ethiopia Demographic and Health Survey [6] result, Neonatal mortality, Infant mortality and Under-5 mortality of Ethiopia declined from 49, 97 and 166 deaths per 1,000 live births in 2000 to 29, 48 and 67 deaths per 1,000 live births in 2016, respectively. This shows that $41 \%, 50 \%$ and $60 \%$ decrease in neonatal, infant and under-5 mortality respectively of Ethiopia over a period of 16 years. In other words, in Ethiopia 1 in every 35 children dies within the first month, 1 in every 21 children dies before celebrating the first birthday, and 1 of every 15 children dies before reaching the fifth birthday. Moreover, residence and regional variation are even more pronounced in the mortality of under-five children [6].

On the other hand, the determinants of child mortality have not been well investigated using recent data in Ethiopia. The objectives of this study were to study the trends of under-five mortality and identify factors associated with under-five mortality in Ethiopia by using data from the 2016 Ethiopia Demographic and Health Survey.

\subsection{Statements of the Problems}

Child mortality has received extensive attention internationally through Sustainable Development Goals (SDGs). Understanding the determinant factors and geographical distribution of under-five child mortality is essential to inform public health policies and design strategies to accelerate the reduction of under-five child mortality. This is particularly important because, under-five child mortality has long been used as one of the measures of the level of socioeconomic developments of a nation, since children are at greater risk of dying before age five. The burden of under-five deaths remains unevenly distributed. About 80 percent of under-five deaths occur in two regions, sub-Saharan Africa and South Asia. Six countries including Ethiopia account for half of the global under-five deaths [32].

For the 5-year period preceding the 2016 survey, the under-five mortality rate was 67 deaths per 1,000 live births which means that 1 in 15 children in Ethiopia dies before reaching age 5 [6]. This rate is still high and it is one of the challenging problems that the country needs to address. Most of the studies made on under-five mortality in Ethiopia are based on more than a decade old data though there are few based on the 2011 EDHS which is about seven years old and extrapolations based on outdated surveys may not be reliable for monitoring changes in health status or for comparative analytical work.

As Ethiopia embarks on the implementation of GTP II and Agenda 2030, investing in reliable and timely data to ensure strong monitoring of the new Sustainable Development Goals is crucial. Thus, this study attempted to explore the major risk factors and assess the regional variation of under-five mortality taking into consideration various health, socio-economic and environmental factors such as mothers age, mothers' education, place of residence, economic status of households, source of drinking water, sanitation, birth interval, breast feeding status, place of birth delivery, etc.

In view of the objectives of the study as well as observations made while reviewing relevant literature, the following research questions have been formulated;

1) Which are the factors that significantly influence under-five child mortality in Ethiopia?

2) Are there significant variations in under-five child mortality across the regional states of Ethiopia?

3) What factor(s) have made significant contribution to the variation of under-five child mortality among regional states of Ethiopia?

\subsection{Objectives of the Study}

General Objective

The main objective of this study was to identify the significant socio-economic and demographic factors influencing under-five child mortality and evaluate the variation of these factors among the regional states of 
Ethiopia using an appropriate multilevel logistic regression model.

Specific Objectives

The specific objectives of the study which should be accomplished to achieve the general objective stated above are:

1) To determine the extent of U5CM within and between regions of Ethiopia.

2) To identify the factors that may explain the variation in U5CM between regions of Ethiopia.

3 ) To carry out trend analysis of under-five mortality during 2000-2016.

\subsection{Significance of the Study}

Under-five mortality rate is one of the most important indicators of the socio-economic well-being and public health conditions of a country. Identifying determinants of under-five mortality (U5M) is important for formulating appropriate health programmes and policies that will help to meet the United nations sustainable development goal (SDG) of reducing under-five mortality to at least as low as 25 deaths per 1,000 live births by 2030 [32]. It can also be used to take more cost-effective interventions and policies to reducing child mortality and to improving the health and life expectancy of the society. Specifically, the finding of this study will:

Help stakeholders in designing, formulation and implementing child health intervention programs and projects for the reduction of child mortality.

provide information about the determinants and trends of under-five mortality to stakeholders like the department of probation and child protection, Ministry of Labour and Social Development, Ministry of Health, National council for children, UNICEF, WHO and other NGO's whose beneficiaries are children so as to improve the quality of child care and their health.

Provide base information to policy makers and researchers that can be used for further studies on under-five child mortality.

Help stakeholders in making informed decision and plan appropriate interventions. This template, created in MS Word 2000/2007/2010 and saved as "Word 97-2000 \& 6.0/95 RTF" for the PC, provides authors with most of the formatting specifications needed for preparing electronic versions of their papers. All standard paper components have been specified for three reasons: (1) ease of use when formatting individual papers, (2) automatic compliance to electronic requirements that facilitate the concurrent or later production of electronic products, and (3) conformity of style throughout a journal publication. Margins, column widths, line spacing, and type styles are built-in; examples of the type styles are provided throughout this document. Some components, such as multi-leveled equations, graphics, and tables are not prescribed, although the various table text styles are provided. The formatter will need to create these components, incorporating the applicable criteria that follow.

\section{Data and Methodology}

\subsection{Source of Data}

The data for this study have been obtained from four consecutive Ethiopia Demography and Health Surveys (EDHSs) conducted in 2000, 2005, 2011 and 2016. The 2000, 2005, 2011 and 2016 Ethiopia Demographic and Health Survey (EDHS) were implemented by the Central Statistical Agency (CSA). By virtue of its mandate, the CSA has conducted the surveys in collaboration with the Federal Ministry of Health (FMoH) and the Ethiopian Public Health Institute (EPHI) with technical assistance from ICF international, and financial as well as technical support from development partners. All actors in this effort have exerted themselves to get reliable, accurate, and up-to-date data to measure the success of the national development agenda-growth and transformation plan II as well as the sustainable development goals. The 2016 survey was conducted from January 18, 2016, to June 27, 2016, based on a nationally representative sample that provides estimates at the national and regional levels and for urban and rural areas.

Ethiopia demographic and health Survey conducted in 2000, 2005, 2011 and 2016, were designed to provide estimate for the Health and Demographic variables of interest for the following domain: Ethiopia as a whole, Urban and rural areas, and 9 geographical administration region (Tigray, Affars, Amhara, Oromiya, Somalia, Benshanguil Gumuz, Southern nationsnationalities and people, Gambela and Harari regional states and 2 city adminstrations: Addis Ababa and Dire Dawa).

Sampling Design of the Survey

The sampling frame used for the 2000, 2005, 2011 and 2016 EDHS data is the Ethiopia Population and Housing Census (EPHC) conducted in 1997 (for the first two surveys) and in 2007 (for the latter two surveys) by the Central Statistical Agency (CSA). The 2016 EDHS sample survey was selected in two stages. Each region was stratified in two rural and urban Areas. Samples of each enumeration area are were selected in independently from each stratum in two stages. From first stage, a total of 645 EAs (202 in urban areas and 443 in rural areas) were selected with probability proportion to EA size. In second stage, a fixed number of 28 household per cluster were selected with random systematic sample selection from the newly created household listening.

A total of 18,008 households were selected for the sample, of which 17,067 were occupied. Of the occupied households, 16,650 were successfully interviewed, yielding a response rate of $98 \%$.

In the interviewed 16583 households, eligible women aged 15-49 were identified for individual interview; complete interviews were 15683, conducted for yielding a response rate of 95 percent. Information for this study was taken from the birth history section of the Women's Questionnaire. The 2016 EDHS data set has hierarchical structure. The hierarchy for this study follows individuals/child as level-1 and regions as level-2. This means that individuals/children are nested in groups/regions.

In this study, the 2000, 2005, 2011 and 2016 EDHS data are 
used to describe the trend of under-five mortality in Ethiopia. The 2016 EDHS data is used to analyze determinants and variation of under-five mortality by background characteristics.

\subsection{Description of the Variables in the Study}

The independent variables that were considered to influence under-five mortality were selected based on findings of available similar studies and the available data on the subject.

\subsubsection{The Response Variable}

The response variable for this study is under-five child mortality. Under-five mortality is defined as the probability of dying before completing the fifth birthday. Thus, the outcome variable is the child event before reaching five years of age, which is dichotomous and coded as 1 if the child died in the five years before the survey and 0 if alive.

In short:

$$
\mathrm{Y}_{\mathrm{i}}=\left\{\begin{array}{c}
1 \text { if the } i^{\text {th }} \text { child died before five years of age } \\
0 \text { if the } i^{\text {th }} \text { under }- \text { five child is still alive }
\end{array}\right.
$$

\subsubsection{Explanatory Variables}

The explanatory variables included in this study are based on the [25] determinates of childhood morbidity and mortality framework for developing countries experience are from the similar studies reviewed. The main indicator for this study variables of under-five child mortality are included the demographic, socioeconomic and environmental factors.

The demographic factors include this study are:

Sex of child $(1=$ male and $2=$ female $)$

Age of child in month

Type of birth $(0=$ Single birth and $1=$ Multiple birth $)$

Birth order number $(1=$ first birth, $2=$ between $2-3,3=$ between $4-5$ and $4=$ above 5 )

Preceding birth interval in month $(0=$ First birth, $1=$ below 24 months, 2=between 24-47 months and $3=$ above 47 months)

Age of mother at first birth ( $1=$ under 20 years, $2=$ between $20-34$ years and $3=35-49$ years)

Family size (Number of HH members) (1= between 1-3, $2=$ between $4-6$ and $3=$ above 6 )

Breastfeeding status $(1=$ ever breastfeed and $2=$ never breast feed).

The socioeconomic variables/factors include:-

Mothers' education level $(0=$ no education, $1=$ primary education, $2=$ secondary education and $3=$ higher education)

Wealth index (economic status of $\mathrm{HH})(1=$ Poor, $2=$ Medium and $3=$ Rich)

Region (1=Tigray, 2=Afar, 3=Amhara, 4=Oromia, 5=Somali, 6=Benshangule-Gumuz, 7=SNNP, 8=Gambella, 9=Harari, 10=Addis Ababa and 11=Diredawa)

Place of residence ( $1=$ urban and $2=$ rural).

And the variables that are classified as environmental variables include:-

Availability of toilet facility $(0=$ improved facility and
$1=$ unimproved facility and $2=$ no toilet facility).

Source of drinking water $(0=$ protected source and $1=$ unprotected source).

Place of delivery $(0=$ at home and $1=$ at health center $)$.

\subsection{Methods of Data Analysis}

In order to study the trend and factors associated with under-five mortality, descriptive statistics, trend analysis, ordinary logistic regression analysis and multilevel logistic regression analysis were used. In the multilevel analysis, three multilevel models (an empty model, model controlling for the individual-level variables, and a model controlling for community-level variables) were constructed.

Logistic regression

Logistic regression is a popular modeling approach when the dependent variable is dichotomous or polytomous. This model allows one to predict outcomes, from a set of variables that may be continuous, discrete, dichotomous, or a mix of any of these. [20] Described logistic regression focusing on its theoretical and applied aspects.

Logistic regression has two main uses:

The first is the prediction of group membership. Since logistic regression calculates the probability of success over the probability of failure, the results of the analysis are in the form of an odds ratio.

Logistic regression also provides knowledge of the relationships and strengths among the variables.

Model description

Binary data are the most common form of categorical data and the most popular model for binary data is logistic regression [2]. Binary logistic regression model is a form of regression, which is used when the dependent variables is dichotomous and also used to investigate the effect of predictors on the probability of having under-five child mortality. The dependent variable is given as

$$
\mathrm{Yij}=\left\{\begin{array}{c}
1 \text { ifchild } i \text { from region } j \text { died before } 5 \text { years of age } \\
0 \text { if the under }- \text { five child is still alive }
\end{array}\right.
$$

$\mathrm{i}=1,2,3 \ldots \mathrm{M}$ and $\mathrm{j}=1,2,3 \ldots \mathrm{N}$

Where: $\mathrm{M}$-is the number of under-five children in each region $\mathrm{j}$ and $\mathrm{N}$-is the number of regions.

The logistic model can be defined in terms of matrix as follows, Let $Y_{n \times 1}$ be a dichotomous outcome random variable with categories 1 (child is dead) and 0 (child is alive) in the five years prior to the survey and $X_{(n \times(k+1))}$ denote the single level binary logistic regression data matrix of k-predictor variables of the under-five child death. Then,

$$
\mathrm{X}=\underbrace{\left(\begin{array}{ccccc}
1 & X_{11} & X_{12} & \ldots & X_{1 k} \\
1 & X_{21} & X_{22} & \ldots & X_{2 k} \\
\cdot & \cdot & \cdot & \ldots & \cdot \\
\cdot & \cdot & \cdot & \ldots & \cdot \\
\cdot & \cdot & \cdot & \ldots & \cdot \\
1 & X_{n 1} & X_{n 2} & \ldots & X_{n k}
\end{array}\right)}_{n \times(k+1)}=\left[\begin{array}{c}
X_{1} \\
X_{2} \\
\cdot \\
\cdot \\
\cdot \\
X_{n}
\end{array}\right], \beta=\left[\begin{array}{c}
\beta_{0} \\
\beta_{1} \\
\beta_{2} \\
\cdot \\
\cdot \\
\cdot \\
\cdot \\
\beta_{k}
\end{array}\right] \sim(\mathrm{K}+1) \mathrm{x} 1
$$

The first (leading) column corresponds to the constant or 
intercept of the logistic regression equation. The matrix $\mathrm{X}$ without the leading column of $1 \mathrm{~s}$, is termed as predictor data matrix and $\beta$ - is the vector of unknown parameters (coefficients of the covariates and intercept).

Then, the conditional probability that the $i^{\text {th }}$ child has died given the vector of predictor variables $X_{i}$ is denoted by $\pi_{i}=\mathrm{P}\left(y_{i}=1 \mid \mathrm{X}_{i}\right)$. The expression $\pi_{i}$ in logistic regression model can be expressed in the form of:

$$
\begin{gathered}
\pi_{i}=\mathrm{P}\left(y_{i}=1 \mid \mathrm{X}_{i}\right)=\frac{\exp \left(\beta_{0}+\beta_{1} x_{i 1}+\cdots \beta_{k} x_{i k}\right)}{1+\exp \left(\beta_{0}+\beta_{1} x_{i 1}+\cdots \beta_{k} x_{i k}\right)}=\frac{\mathrm{e}^{\mathrm{X}_{i} \beta}}{1+\mathrm{e}^{\mathrm{X}_{i} \beta}}, \\
\mathrm{i}=1,2,3 \ldots \ldots \mathrm{n}
\end{gathered}
$$

Where $\pi_{i}$ is the probability that the $i^{\text {th }}$ child dies before five years of age given the vector of predictors $\left(X_{i}\right)$.

Assumption of Binary Logistic Regression

As indicated in the above sections, the advantage of the logistic regression is that it has flexible assumptions as compared with discriminant analysis. There are, however, other assumptions one should consider for the efficient use of logistic regression as detailed in [19].

The Odds of Ratio

The odds ratio is defined as the ratio of the odds that the event occurs (success) to the odds that the event will not occur (failure). In binary logistic regression analysis, odds ratio is the exponent of the estimated coefficient, $\exp (\hat{\beta})$.

In logistic regression analysis, it is assumed that the explanatory variables affect the response through a suitable transformation of the probability of the success. This transformation is a suitable link function of $\pi_{i}$, and is called the logit-link, which is defined as:

$\log i t\left(\pi_{i}\right)=\log \left(\frac{\pi_{i}}{1-\pi_{i}}\right)=\beta_{0}+\beta_{1} x_{i 1}+\cdots \beta_{k} x_{i k}=\sum_{j=0}^{k} \beta_{j}{ }^{\prime} \mathrm{X}_{i j}$

where, $i=1,2, \ldots n ; j=0,1, \ldots, k$

Each coefficient of a continuous covariate is interpreted as the change in the expected log-odds of having child death before five years of age per unit change of the covariate. In case of categorical predictor variable, it is interpreted as the log-odds of having child death before five years of age with a given category compared to the reference category [8].

Estimation of Logistic Regression Parameter

The most commonly used method for estimating the parameters of a logistic regression model is the method of Maximum Likelihood (ML) estimation. The method of maximum likelihood estimation yields to estimate values for the unknown parameters which maximize the probability of obtaining the observed set of data. For this study to estimate of the parameter of the logistic regression model by using the estimate of the maximum likelihood equation.

Suppose $(\mathrm{y} 1, \mathrm{y} 2, \mathrm{y} 3, \ldots . . \mathrm{yn})$ represent the $\mathrm{n}$ dependent random observation corresponding to the $\mathrm{Yi}$ is a bernoulli random variables. The probability function of $\mathrm{Yi}$ is

$$
\mathrm{f}\left(\mathrm{y}_{\mathrm{i}}\right)=\pi^{y i}(1-\pi)^{1-y i} ; \mathrm{y}_{\mathrm{i}}=0 \text { or } 1 \text { and logistic model is }
$$$$
\pi_{i}=\mathrm{P}\left(y_{i}=1 \mid \mathrm{X}_{i}\right)=\frac{\mathrm{e}^{\mathrm{x}_{i} \beta}}{1+\mathrm{e}^{\mathrm{X}_{i} \beta}} \mathrm{i}=1,2,3 \ldots . . \mathrm{n} \text {. Since } \mathrm{Y} \text { 's are }
$$

assumed to be independent, the joint probability function or likelihood function is given by:

$$
\begin{aligned}
L(\beta)=\mathrm{L} & \left(\beta_{0}, \beta_{1}, \beta_{2}, \beta_{3}, \ldots \ldots, \beta_{\mathrm{k}}\right)=\prod_{i=1}^{n} \pi^{y i}(1-\pi)^{1-y i} \\
& =\prod_{\mathrm{i}=1}^{\mathrm{n}}\left[\frac{\mathrm{e}^{\mathrm{x}_{i} \beta}}{1+\mathrm{e}^{\mathrm{x}_{i} \beta}}\right]^{\mathrm{y}_{\mathrm{i}}}\left[\frac{1}{1+\mathrm{e}^{\mathrm{x}_{i} \beta}}\right]^{1-\mathrm{y}_{\mathrm{i}}}
\end{aligned}
$$

Where, $L(\beta)$ is the likelihood function. The maximum likelihood estimates of the parameters $\beta$ are obtained by taking the $\log$ of the likelihood function and maximizing the log-likelihood function which is given by:-

$$
\log L(\beta)=\sum_{i=1}^{n}\left\{y_{i} \log \left[\frac{\mathrm{e}^{\mathbf{x}_{i} \mathbf{\beta}}}{1+\mathrm{e}^{\mathbf{x}_{i} \mathbf{\beta}}}\right]+\left(1-\mathrm{y}_{i}\right) \log \left[\frac{1}{\left.1+\mathrm{e}^{\mathbf{x}_{i}}\right]}\right]\right\}
$$

Differentiating equation (6), the log likelihood function with respect to each $\beta$ and setting each equation equal to zero, we can theoretically obtain estimate of the vector of parameters $\beta$. But the equation is nonlinear in $\beta$, and as a result the estimates do not have a closed form expression. Therefore, $\beta$ will be obtained by maximizing log-likelihood using iterative algorithm method [3].

\subsection{Goodness-of-Fit of the Model}

The goodness of fit of a model measures how effective or well the model describes the response variable. Assessing goodness of fit involves investigating how the predicted values are closer to the observed values. Clearly, the fit is good if there is a good agreement between the fitted and the observed data. Some common approaches to test the goodness of fit the model are Pearson's $\chi^{2}$ statistic (Hosmer and Lemeshow Test Statistic), Wald test and the likelihood-ratio test [1].

\subsubsection{The Hosmer-Lemeshow Test}

The Hosmer-Lemeshow test is one of the common recommended tests for the goodness of fit of a binary logistic regression model. It is based on dividing the data into $g$ ( $g$ is usually taken as 10) equal groups and then comparing the number actually observed in each group to the number expected. Using this grouping strategy, the Hosmer-Lemeshow goodness-of- fit statistic, $\hat{\mathrm{C}}$ is obtained by calculating the Pearson chi-square statistic from the $g \times 2$ Table of observed and estimated expected frequencies. A formula defining the calculation of $\hat{\mathrm{C}}$ is as follows:

$$
\hat{C}=\frac{\sum_{j}^{g}\left(O_{j}-E_{j}\right)^{2}}{V_{j}} \text { Where } E_{j}=n \pi_{j},
$$
$V_{j}=n \pi_{j}\left(1-\pi_{j}\right), \mathrm{g}$ is the number of groups, $O_{j}$ is observed number of events in the $j^{\text {th }}$ group, $E_{j}$ is expected number of events in the $j^{\text {th }}$ group, and $V_{j}$ is a variance correction factor for the $j^{\text {th }}$ group. If the observed number of events differs from what is expected by the model, the statistic $\hat{c}$ will be large and there will be evidence against the null hypothesis that the model is adequate in fitting the data. This statistic has an approximate chi-square distribution with $(g-2)$ degrees of freedom [1]. 
If the calculated p-value of the Hosmer-Lemeshow goodness-of-fit test statistic is greater than 0.05 , we will not reject the null hypothesis that there is no difference between observed and model-predicted values, implying that the model estimates are adequate to fit the data at an acceptable level.

\subsubsection{The Likelihood Ratio Test}

The likelihood ratio chi-square $\left(G^{2}\right)$ statistic is the test statistic commonly used for assessing the overall fit of the logistic regression model. The likelihood ratio test, also called log-likelihood test, is based on -2LL ( -2 times log likelihood). The likelihood ratio statistic is obtained by subtracting two times log likelihood (-2LL) for the final (full) model from the $\log$ likelihood for the intercept only model. This $\log$ likelihood-ratio test uses the ratio of the maximized value of the likelihood function for the intercept only model $\mathrm{L}_{0}$ over the maximized value of the likelihood function for the full model $\mathrm{L}_{1}$. The likelihood test statistic is given by:

$$
\mathrm{G}^{2}=-2 \ln \left[\frac{L_{0}}{L_{1}}\right]=-2\left\{\ln L_{0}-\ln L_{1}\right\}=-2\left[L L_{0}-L L_{1}\right]
$$

Where $L L_{0}$ is the log likelihood value of the model which have the intercept term only and $L L_{1}$ is the log likelihood value of the full model, where $L_{0}$ is the likelihood function of the null model and $L_{1}$ is the likelihood function of the full model evaluated at the MLEs.

The likelihood ratio statistic has a chi-square distribution and it tests the null hypothesis that all logistic regression coefficients except the constant are zero. The degrees of freedom are obtained by differencing the number of parameters in the two models.

A p-value is less than $5 \%$ leads to the rejection of the null hypothesis that all the predictor effects are zero. When this likelihood test is significant, at least one of the predictors is significantly related to the response variable.

Under the global null hypothesis, $\mathrm{H} 0: \beta 1=\beta 2=\ldots=\beta \mathrm{k}=0$, the statistic G2 follows a chi-square distribution with $k-1$ degrees of freedom and measures how well the independent variables affect the response variable [20].

\subsubsection{The Wald Test}

The Wald statistics is used to test the significance of individual logistic regression coefficient for each independent variables, that is to test the parameters associated with an explanatory variables is zero or not. If the parameter of an explanatory variable is significantly different from zero then the associated variable should be included in the model. Wald test statistic has a chi-square distribution with one degree of freedom, and used to test the significance of individual coefficients in the model and the hypothesis to be tested is: $H_{0}: \quad \beta j=0$ against $\mathrm{H}_{1}: \beta j \neq 0, \mathrm{j}=1 \ldots \mathrm{k}$ at $\alpha$ level of significance.

The Wald test statistic, $Z^{2}$ for this hypothesis is

$$
Z^{2}=\left[\frac{\beta_{j}}{S E\left(\beta_{j}\right)}\right]^{2} \sim \chi^{2}(1)
$$

\subsection{Multilevel Logistic Regression Model}

A multilevel logistic regression model also referred to in the literature as a hierarchical model, can account for lack of independence across levels of nested data (e.g., children nested within regions). Standards logistic regression assumes that all experimental units (in this case, under-five children) are independent in the sense that any variables affecting the dependent variable have the same effect in all regions. Multilevel modeling relaxes this assumption and allows these variables' effects to vary across regions. Because of cost, time and efficiency considerations, stratified multistage samples are the norm for sociological and demographic surveys.

For such samples the clustering of the data is, in the phase of data analysis and data reporting, a nuisance which should be taken into consideration.

This clustering sampling scheme often introduces multilevel dependency or correlation among the observations that can have implications for model parameter estimates. For multistage clustered samples, the dependence among observations often comes from several levels of the hierarchy. The problem of dependencies between individual observations also occurs in survey research, where the sample is not taken randomly but cluster sampling from geographical areas is used instead. In this case, the use of single-level statistical models is no longer valid and reasonable. Hence, in order to draw appropriate inferences and conclusions from multistage stratified clustered survey data, we may require tricky and complicated modeling techniques like multilevel modeling [21].

In this study, multilevel binary logistic regression model would be adopted to uncover the under-five child mortality variations among regional states of Ethiopia.

\subsubsection{A Two-Level Logistic Regression Model}

Multilevel models are statistical models which allow not only independent variable at any level of hierarchical structure but also at least one random effect above level one group [13]. A multilevel logistic regression model can account for lack of independence across levels of nested data (i.e., individuals nested within regions). Conventional logistic regression assumes that all experimental units are independent in the sense that any variable which affects occurrence of death has the same effect in all regions, but multilevel models are used to assess whether the effect of predictors vary from region to region.

The multilevel binary logistic regression model has a binary outcome (child died or is alive). In this study the basic data structure of the two-level logistic regression is a collection of $N$ groups (regions) and within-group $j(j=1,2, \ldots, N)$, a random sample $n_{j}$ of level-one units (children). We let the response variable $Y_{i j}=1$ if the $i^{\text {th }}$ under five child in region $\mathrm{j}$ has died, and $\mathrm{Y}_{i j}=0$ otherwise; with probabilities, $\pi_{i j}=$ $\mathrm{P}\left(y_{i j}=1 \mid \mathrm{X}_{i j}, u_{j}\right)$, the probability that the $\mathrm{i}^{\text {th }}$ child from region $\mathrm{j}$ died and $1-\pi_{i j}=\mathrm{P}\left(y_{i j}=0 \mid \mathrm{X}_{i j}, u_{j}\right)$, the probability that the $\mathrm{i}^{\text {th }}$ child from region $\mathrm{j}$ is alive; where $u_{j}$ is a random effect and often assumed to be $N\left(0, \sigma_{o}^{2}\right)$. 
The standard assumption is that $\mathrm{Y}_{i j}$ has a Bernoulli distribution. Let $\pi_{i j}$ be modeled using a logit link function. The two-level model is given by:

$$
\operatorname{logit}\left(\pi_{\mathrm{ij}}\right)=\log \left(\frac{\pi_{i j}}{1-\pi_{i j}}\right)=\beta_{o j}+\sum_{l=1}^{k} \beta_{l j} x_{l i j} ; l=1,2,, k \ldots . .
$$

Where $\quad \beta_{0 j}=\beta_{0}+U_{0 j}, \beta_{1 j}=\beta_{1}+U_{1 j}, \ldots, \beta_{k j}=\beta_{k}+$ $U_{k j}$ The level-two model (3.7) can be rewritten as:

$$
\operatorname{logit}\left(\pi_{\mathrm{ij}}\right)=\log \left(\frac{\pi_{i j}}{1-\pi_{i j}}\right)=\beta_{o}+\sum_{l=1}^{k} \beta_{l} x_{l i j}+U_{o j}+\sum_{l=1}^{k} U_{l j} x_{l i j} \ldots .
$$

Where $\mathrm{X}_{i j}=\left(\mathrm{X}_{1 i j}, \mathrm{X}_{2 i j}, \ldots, \mathrm{X}_{k i j}\right)$ represent the covariates, $\beta=\left(\beta_{0}, \beta_{1}, \ldots, \beta_{\mathrm{k}}\right)$ are regression coefficients, $U_{0 j}, U_{1 j}, \ldots, U_{k j}$ are the random effects of model parameter at level two. It is assumed that the $U_{0 j}, U_{1 j}, \ldots, U_{k j}$ follow a normal distribution with mean zero and variance $\delta_{o}^{2}$. Without $U_{0 j}, U_{1 j}, \ldots, U_{k j}$, equation (8) can be considered as a single level logistic regression model. Therefore, conditional on $U_{0 j}, U_{1 j}, \ldots, U_{k j}$, the $y_{i j}$ can be assumed to be independently distributed as Bernoulli random variables ( $\mathrm{Li}$ et al., 2011).

Heterogeneity of Proportion

Consider a population having two-levels. A random sample of $\mathrm{n}_{\mathrm{j}}$ level-one (children) units is collected from level two groups (region) $(j=1,2, \ldots, N)$. The outcome variable is dichotomous and denoted by $\mathrm{Y}_{i j}\left(i=1,2, \ldots, n_{j} ; j=\right.$ $1,2, \ldots, N)$ for level-one unit $i$ nested in level-two group $j$. The total sample size is $M=\sum_{j=1}^{N} n_{j}$. If one does not (yet) take any explanatory variable into account, the probability of success is constant in each group $\mathrm{j}$, denoted by $\pi_{j}$. The dichotomous outcome variable for the child $\mathrm{i}$ in region $j, Y_{i j}$ can be expressed as the sum of the probability in $\mathrm{j}$ region $\pi_{j}$ (the average proportion of I levels in region $\left.j, \mathrm{E}\left(Y_{i j}\right)=\pi_{j}\right)$ plus some individual dependent residual, that is

$$
y_{i j}=\pi_{j}+\varepsilon_{i j}
$$

The residual term is assumed to have mean zero and variance, $\operatorname{Var}\left(\varepsilon_{i j}\right)=\pi_{j}\left(1-\pi_{j}\right)$ Since the outcome variable is coded 0 and 1 , the group (region) sample average is the proportion of successes in group $\mathrm{j}$ given by:

$$
\widehat{\pi}_{J}=\frac{1}{n_{j}} \sum_{i=1}^{n_{j}} Y_{i j}
$$

Where $\hat{\pi}_{J}$ is an estimate for the group-dependent probability $\pi_{j}$. Similarly, the overall sample average is the overall proportion of success, $\pi$ and given by:$\pi=\frac{1}{M} \sum_{j=1}^{N} \sum_{i=1}^{n_{j}} Y_{i j}$

Testing Heterogeneity of Proportions

For the proper application of multilevel analysis the first logical step is to test for the heterogeneity of proportions of under-five child mortality between groups (in our case between regions). To test whether there are indeed systematic differences between groups, the well-known chi-square test for contingency table is often given in the familiar form
$\sum(O-E)^{2} / E$ where $O$ is the observed and $E$ the expected count in a cell of the contingency table. In this case it can be written also as

$$
\chi 2=\sum_{j=1}^{N} n_{j} \frac{\left(\pi_{j}-\widehat{\pi}\right)^{2}}{\widehat{\pi}(1-\widehat{\pi})}
$$

This statistic follows approximately chi-square distribution with $N-1$ degrees of freedom. The approximation is valid if the expected numbers of success and of failures in each group, $n_{j} \pi_{j}$ and $n_{j}\left(1-\pi_{j}\right)$, respectively, are at least 1 while 80 percent of them are at least 5 [1]. This condition will not always be satisfied, and the chi-square test then may be seriously in error. For a large number of groups the null distribution of the test statistic of the chi-square can be approximated by a normal distribution with the correct mean and variance [24].

Estimation of between and within group variance: the theoretical (true) variance between the group dependent probabilities, i.e., the population value of $\operatorname{var}\left(\pi_{j}\right)$, can be estimated by: $\hat{\tau}^{2}=S_{\text {between }}^{2}-\frac{S_{\text {wthin }}^{2}}{\tilde{n}} \quad$ where

$$
\tilde{n}=\frac{1}{N-1}\left(M-\frac{\sum_{j=1}^{N} n_{j}^{2}}{M}\right)
$$

For dichotomous outcome variables, the observed between group variance is closely related to the chi-square test statistic given in equation (13).

Between group variance

$$
S_{\text {between }}^{2}=\frac{\hat{\pi}(1-\hat{\pi})}{\tilde{n}(N-1)} \chi^{2} \text {, (13) where } \chi^{2} \text { is as given by eqn }
$$

(13), and the within group variance in case of a dichotomous outcome variable is a function of group averages which is given by:

$$
S_{\text {within }}^{2}=\frac{1}{M-N} \sum n_{j} \pi_{j}\left(1-\pi_{j}\right) .
$$

\subsubsection{The Empty Logistic Regression Model}

The empty two-level model for a dichotomous outcome variable refers to a population of groups (level-two units) and specifies the probability distribution for group-dependent probabilities $\pi_{j}$ (probability of having $i^{\text {th }}$ child in $j^{\text {th }}$ group (region) dead before five year of age). Consider equation (8) without taking further explanatory variables into account. We focus on the model that specifies the transformed probabilities $f\left(\pi_{j}\right)$ to have a normal distribution. This is expressed, for a general link function $\mathrm{f}(\pi)$, by the formula,

$$
f\left(\pi_{j}\right)=\beta_{o}+U_{o j}
$$

Where $\beta_{o}$ is the population average of the transformed probabilities and $U_{o j}$ is the random deviation from this 
average for group $\mathrm{j}$. If $\mathrm{f}(\pi)$ is the logit function, then $f\left(\pi_{j}\right)$ is just the log-odds for group $\mathrm{j}$. Thus, for the logit link function, the log-odds have a normal distribution in the population of groups, which is expressed by:

$$
\operatorname{logit}\left(\pi_{j}\right)=\beta_{o}+U_{o j}
$$

For the derivation of $U_{o j}$ are independent random variables with normal distribution of with mean zero and variance $\sigma_{0}^{2}$. This is because the individual (level one) residual variance of the dictums outcome variables follows directly from the success probability of the $y_{i j}$ (death or alive of under-five child follows Bernoulli distribution direct from the probability of having under five child death $\left(\pi_{j}\right)$ ) which is given by: $\operatorname{Var}\left(\varepsilon_{i j}\right)=$ $\pi_{j}\left(1-\pi_{j}\right)$ denoted by $\pi_{0}$ the probability corresponding to the average value $\beta_{o}$ as defined as: $f\left(\pi_{0}\right)=\beta_{0}$

For the logit function, the so-called logistic transformation of $\beta_{0}$, is defined by

$$
\pi_{0}=\operatorname{logistic}\left(\beta_{0}\right)=\frac{\exp \left(\beta_{0}\right)}{1+\exp \left(\beta_{0}\right)}
$$

Note that due to the non-linear nature of the logit link function, there is no a simple relation between the variance of probabilities and the variance of the deviations $U_{0 j}$ (Snijders and Bosker, 2012). An approximate variance of the probability is given by:

$$
\operatorname{var}\left(\pi_{j} \approx\left(\pi_{0}\left(1-\pi_{0}\right)\right)^{2} \sigma_{0}^{2}\right)
$$

\subsubsection{The Random Intercept Logistic Regression Model}

In the random intercept logistic regression model the intercept is the only random effect meaning that the groups differ with respect to the average value of the response variable. But the relation between explanatory and response variables can differ between groups in more ways. It represents the heterogeneity between groups in the overall response.

Then, random intercept model expresses the log-odds, i.e. the logit of $\pi_{\mathrm{ij}}$, as a sum of a linear function of the explanatory variables (all indicators of having under-five child mortality). That is,

$$
\begin{gathered}
\operatorname{logit}\left(\pi_{i j}\right)=\log \left[\frac{\pi_{i j}}{1-\pi_{i j}}\right]=\beta_{0 j}+\beta_{1} x_{1 i j}+\beta_{2} x_{2 i j}+\ldots+ \\
\beta_{k} x_{k i j}=\beta_{0 j}+\sum_{h=1}^{k} \beta_{h} x_{h i j}
\end{gathered}
$$

Where, $\operatorname{logit}\left(\pi_{i j}\right)$ does not include a level-one residual because it is an equation for the probability of having under-five child death $\left(\pi_{i j}\right)$ rather than for the outcome $Y_{i j}$, the intercept term $\beta_{0 j}$ is assumed to vary randomly and is given by the sum of an average intercept $\beta_{0}$ and group-dependent deviations $u_{o j}$ that is $\beta_{0 j}=\beta_{0}+u_{o j}$ and on substituting in the above equation, we get.

$$
\begin{gathered}
\operatorname{logit}\left(\pi_{i j}\right)=\beta_{0}+\sum_{h=1}^{k} \beta_{h} x_{h i j}+u_{0 j} \\
\text { Solving for } \pi_{\mathrm{ij}}, \pi_{i j}=\frac{e^{\beta_{0}+\sum_{h=1}^{k} \beta_{h} x_{h i j}+u_{0 j}}}{1+e^{\beta_{0}+\sum_{h=1}^{k} \beta_{h} x_{h i j}+u_{0 j}}}
\end{gathered}
$$

Where from equation (20), $\beta_{\mathrm{o}}+\sum_{\mathrm{h}=1}^{\mathrm{k}} \beta_{\mathrm{h}} \mathrm{x}_{\mathrm{hij}}$ is the fixed part of the model. $u_{0 j}$ is called the random part of the model. $U_{o j}$ are independent random variables with normal distribution of with mean zero and variance $\sigma_{0}^{2}$.

Random intercept models have many applications, for instance estimating the regional effects on under-five child mortality, adjusting for individual child's level factors, and within the model, evaluate and compare the performance of the region's under-five child mortality reduction. This can be done by obtaining the odds ratio for each region. This regional effect is a measure of the situation of under-five child mortality due to the region relative to the average of all regions. If the odd of under-five child mortality for regional effects is sufficiently larger than one, the region is considered to have performed worse than the average; if it is significantly smaller than one, the region is considered to have better performance than the expected [9].

\subsubsection{The Random Coefficient Logistic Regression Model}

In logistic regression and linear model analysis are constructed for the log-odds. The multilevel approach the random coefficient logistic regression is based on linear models for the log-odds that include random effects for the groups or other higher level units. The success probability is not necessarily the same for all individuals in a given group. Therefore the success of probability depends on the individual or the group. It is denoted by $\pi_{i j}$.

Now $\log i t\left(\pi_{i j}\right)$, on a single level one explanatory variable $\mathrm{X}$,

$$
\operatorname{logit}\left(\pi_{\mathrm{ij}}\right)=\log \left(\frac{\pi_{i j}}{1-\pi_{i j}}\right)=\beta_{o j}+\beta_{1 j} x_{1 i j}
$$

The intercepts $\beta_{\mathrm{oj}}$ as well as the regression coefficients or slopes, $\beta_{1 \mathrm{j}}$ are group (region) dependent. These group dependent coefficients can be split into an average coefficient and the group dependent deviation:

$\beta_{o j}=\beta_{o}+U_{o j}, \beta_{1 j}=\beta_{1}+U_{1 j}$ Thus, by Substitution into (22), we get

$$
\begin{gathered}
\operatorname{logit}\left(\pi_{\mathrm{ij}}\right)=\log \left(\frac{\pi_{i j}}{1-\pi_{i j}}\right)=\left(\beta_{o}+U_{o j}\right)+\left(\beta_{1}+U_{1 j}\right) x_{1 i j}= \\
\beta_{o}+\beta_{1} x_{1 i j}+U_{o j}+U_{1 j}
\end{gathered}
$$

Then, we have two random group effects: - the random intercept $U_{o j}$ and the random slope $U_{1 j}$. It is assumed that the level two residuals $U_{o j}$ and $U_{1 j}$ have both zero mean given the value of the explanatory variable and the variance are denoted by $\sigma_{0}{ }^{2}, \sigma_{1}{ }^{2}$ and their covariance is $\sigma_{01}$. Thus, $\beta_{1}$ is the average regression coefficient given explanatory variable $x_{1}$ like $\beta_{0}$ is the average intercept of the response variable. The first part of equation (23), $\beta_{\mathrm{o}}+\beta_{1} \mathrm{x}_{1 \mathrm{ij}}$ is called the fixed part of the model whereas the second part $U_{o j}+U_{1 j} x_{1 i j}$ is called the random part of the model.

The term $U_{o j}+U_{1 j} X_{1 i j}$ can be regarded as a random interaction between group and predictors (X). This model implies that the groups are characterized by two random effects: their intercept and their coefficient. These two 
groups effects $U_{o j}$ and $U_{1 j}$ will not be independent (are correlated). Further, it is assumed that, for different groups, the pairs of random effects $\left(U_{o j}, U_{1 j}\right)$ are independent and identically distributed. Thus, the variances and covariance of the level-two random effects $\left(\mathrm{U}_{\mathrm{oj}}, \mathrm{U}_{1 \mathrm{j}}\right)$ are denoted by:

$$
\begin{gathered}
\operatorname{Var}\left(U_{o j}\right)=\sigma_{00}=\sigma_{0}{ }^{2}, \operatorname{Var}\left(U_{1 j}\right)=\sigma_{11}=\sigma_{1}{ }^{2} \text { and } \\
\operatorname{Cov}\left(U_{o j}, U_{1 j}\right)=\sigma_{01}
\end{gathered}
$$

The above discussion for a single explanatory variable can be extended by including more variables that have random effects. Suppose that there are $\mathrm{k}$ level-one explanatory variables $\mathrm{X}_{1}, \mathrm{X}_{2}, \ldots, \mathrm{X}_{\mathrm{k}}$, and consider the model where all predictor variables have varying slopes and random intercept. That is

$$
\operatorname{logit}\left(\pi_{\mathrm{ij}}\right)=\log \left(\frac{\pi_{i j}}{1-\pi_{i j}}\right)=\beta_{o j}+\beta_{1 j} x_{1 i j}+\beta_{2 j} x_{2 i j}+\cdots+\beta_{k j} x_{k i j}
$$

Letting $\beta_{\mathrm{oj}}=\beta_{\mathrm{o}}+\mathrm{U}_{\mathrm{oj}}$ and $\beta_{\mathrm{hj}}=\beta_{\mathrm{h}}+\mathrm{U}_{\mathrm{hj}} \quad$ where $\mathrm{h}=1,2, \ldots, \mathrm{k}$, we have:

$$
\begin{gathered}
\operatorname{logit}\left(\pi_{\mathrm{ij}}\right)=\log \left(\frac{\pi_{i j}}{1-\pi_{i j}}\right)=\beta_{o}+\sum_{h=1}^{k} \beta_{h} x_{h i j}+U_{o j} \\
+\sum_{h=1}^{k} U_{h j} x_{h i j}
\end{gathered}
$$

Where, the first part $\beta_{\mathrm{o}}+\sum_{\mathrm{h}=1}^{\mathrm{k}} \beta_{\mathrm{h}} \mathrm{x}_{\mathrm{hij}}$ is called the fixed part of the model, and the second part, $\mathrm{U}_{\mathrm{oj}}+\sum_{\mathrm{h}=1}^{\mathrm{k}} \mathrm{U}_{\mathrm{hj}} \mathrm{x}_{\mathrm{hij}}$ is called the random part of the model. The random variables or effects, $U_{0 j}, U_{1 j}, \ldots, U_{k j}$ are assumed to be independent between groups but may be correlated within groups. So the components of the vector $\left(U_{0 j}, U_{1 j}, \ldots, U_{k j}\right)$ are independently distributed as a multivariate normal distribution with zero mean vector and variances and co-variances matrix $\Omega$ given by:

$$
\Omega=\left(\begin{array}{cccc}
\sigma_{0}^{2} & & \cdots & \cdot \\
\sigma_{01} & \sigma_{1}^{2} & \cdots & . \\
\vdots & \vdots & \ddots & \vdots \\
\sigma_{0 k} & \sigma_{1 k} & \cdots & \sigma_{k}^{2}
\end{array}\right)
$$

Intra-Class Correlation Coefficient (ICC)

The other fundamental reason of using multilevel analysis is the existence of intra-class (intra-regional) correlation arising from similarity of child mortality in the same region compared to those of different regions. The intra-class correlation coefficient (ICC) measures the proportion of variance in the outcome explained by the grouping structure. ICC can be calculated using an intercept-only model. This model can be derived from equation (20) by excluding all explanatory variables, which results in the following equation: $\left(\operatorname{logit}\left(\pi_{\mathrm{j}}\right)=\beta_{o+} U_{o j}\right)$. The ICC is then calculated based on the following formula:

$$
I C C=\frac{\delta_{o}^{2}}{\delta_{o}^{2}+\delta_{e}^{2}}
$$

where $\delta_{e}^{2}$ variance of individual (lower) level units and $\delta_{o}^{2}$ a variation due to regional level. In multilevel logit model level one residual variance $\delta_{e}^{2}=\frac{\pi^{2}}{3} \approx 3.29$ (Snijders and Bosker, 1999 ) this formula can be reformulated as:

$$
I C C=\frac{\delta_{o}^{2}}{\delta_{o}^{2}+3.29}
$$

\subsubsection{Estimation and Testing Technique for Multilevel Logistic Model}

The estimation methods of most of the statistical models are well established. But this is not true for multilevel models for binary data. Parameter estimation for multilevel logistic model is not straightforward like the methods for simple logistic regression models. The most common methods for estimating multilevel logistic regression model are Marginal Quasi Likelihood and Penalized Quasi Likelihood which are the two more approximately procedures. There are different methods of parameter estimations which are implemented by various software packages such as, MLwiN, SPSS, STATA and SAS. In this study, the multilevel data were analyzed by the SPSS and STATA software packages.

\subsubsection{Multilevel Model Selection Criteria}

There are generally many options available when modeling a data structure. For this model comparison were used Akakie Information Criteria (AIC) and Bayesian Information Criteria (BIC). It is given as below.

$$
\mathrm{AIC}=-2 \ln (\mathrm{L}(\text { Model }))+2 \mathrm{k}
$$

Bayesian Information Criterion (BIC)

It is also known as the Schwarz criterion after Gideon Schwarz and virtually identical to the minimum description length criterion (Taper, 2004). The formula is given as:

$$
\mathrm{BIC}=-2 \ln (\mathrm{L}(\text { Model }))+\mathrm{k}^{*} \ln (\mathrm{n})
$$

Where: $\mathrm{k}$ is number of estimated parameters, $\mathrm{L}$ (model) is the likelihood of the model $n$ - is number of observation based on the model selection criterion stated above. The model with smallest AIC and BIC value is considered as a better fit model.

Details of multilevel logistic regression models are provided by [12].

\section{Statistical Data Analysis and Results}

In this chapter, results of our analysis are presented. The 2016 EDHS survey data were analyzed with the help of SPSS version 20 and STATA version 13 statistical (software) packages. The results of our analyses (descriptive, single level binary logistic regression and multilevel logistic regression) are presented in three sections of the current chapter.

\subsection{Results of Descriptive Analysis}

The distribution of under-five child mortality in Ethiopia corresponding to the socio-economic, demographic and environmental characteristics is presented in Table 1 . The total number of children aged 0 to 59 month covered in the present study is 10,641 .

Of the total number of children included in the study, $48 \%$ 
were female. As displayed in table 1, the rate or proportion of under-five child mortality varied from one Region to another with the highest proportion (8.5\%) of U5CM recorded in Afar followed by Benishangul-Gumuz (7.3\%) and Somali \& Harari (6.8\%). However, the least proportion of under-five child mortality was observed in Addis Ababa (3.0\%) followed by Tigray (4.0\%) and Amhara. Hence, there appears to be some variation in the proportion of U5CM among the regions of Ethiopia.

The proportion of under-five child mortality occurred more among males than among females. The prevalence of U5CM among females and males were $5.0 \%$ and $6.9 \%$, respectively. The chi-square test results showed a significant association between U5CM and gender ( $\mathrm{p}=0.000)$.

Under-five child mortality in urban areas was also different from the rate in rural areas. Table 1 shows that the proportion of under-five child mortality in urban areas was $3.4 \%$ while it was $6.6 \%$ in rural areas. This shows that the rate of under-five child mortality in rural areas was about 1.94 times higher than the rate in urban areas. Conversely, the proportion of under-five mortality by birth type indicated unexpected variation. A higher percentage (20.9\%) of death of under-five children was observed in multiple birth categories as compared to the $5.6 \%$ death of under-five children observed in single birth categories.

Table 1 shows that the birth order of $6.7 \%$ of the children who died was five or more. Similarly, $6.3 \%$ of the children that died had first birth order, $5.9 \%$ had birth order 4 and 5 while $5.3 \%$ were of birth order 2 and 3 .

The number of death of under- five children also varied according to household size and age of mothers at first birth. Number of household members indicated unexpected variation of under-five child mortality. Contrary to our expectation, a higher percentage $(11.0 \%)$ of death of under-five children was observed in households of size three or less and the lowest percentage $(4.6 \%)$ of death of under-five children was observed in households of size seven or more. On the other hand, a death of about $6.2 \%$ was observed among children born to mothers whose age at first birth was under 20 while none of the children born from mothers in the age group 35-49 died.

Breast feeding was important for the survival of under-five children. As expected, the highest percentage (33.2\%) of never breast fed under-five children died while mothers who ever breastfed lost only $4.4 \%$ of their children.

The prevalence of death of under-five children varied by mothers' level of education. Table 1 reveals that the highest percentage $(6.6 \%)$ of death of under-five children was observed among mothers' with no education as opposed to the lowest percentage $(1.8 \%)$ of death of under-five children recorded among mothers with higher education.

Children born to poor families had highest proportion of mortality $(6.9 \%)$ while children born to rich families had the least proportion of under-five child mortality (4.6\%).

The experiences of under-five child mortality were different by the source of drinking water and type of toilet facility. A higher proportion $(6.9 \%)$ of mortality was recorded among children that drunk water from unprotected sources and a relatively less prevalence $(5.3 \%)$ of mortality were observed among under-five children that drunk water from protected sources. Similarly, a higher proportion $(6.9 \%)$ of under-five children died in households with no toilet facility while it was (3.4\%) of among children from households having improved toilet facility.

Table 1. The distribution of U5CM in Ethiopia by socio-economic, demographic and environmental factors: 2016 EDHS data.

\begin{tabular}{|c|c|c|c|c|c|c|c|}
\hline \multirow{2}{*}{ Variable } & \multicolumn{4}{|c|}{ Under-five child's mortality status } & \multirow{2}{*}{ Chi-square } & \multirow{2}{*}{ Df. } & \multirow{2}{*}{ p-value } \\
\hline & Alive & Dead & $\%$ of U5CM & Total & & & \\
\hline \multicolumn{8}{|l|}{ Sex of child } \\
\hline Male & 5107 & 376 & $6.9 \%$ & 5483 & \multirow{3}{*}{15.970} & \multirow{3}{*}{1} & \multirow{3}{*}{$.000 * *$} \\
\hline Female & 4899 & 259 & $5.0 \%$ & 5158 & & & \\
\hline Total & 10006 & 635 & $6.0 \%$ & 10641 & & & \\
\hline \multicolumn{8}{|l|}{ Birth type } \\
\hline single birth & 9786 & 577 & $5.6 \%$ & 10363 & \multirow{2}{*}{112.876} & \multirow{2}{*}{1} & \multirow{2}{*}{$.000 * *$} \\
\hline multiple birth & 220 & 58 & $20.9 \%$ & 278 & & & \\
\hline \multicolumn{8}{|c|}{ Birth order number } \\
\hline First & 2031 & 136 & $6.3 \%$ & 2167 & \multirow{4}{*}{5.762} & \multirow{4}{*}{3} & \multirow{4}{*}{.124} \\
\hline between $2-3$ & 3162 & 176 & $5.3 \%$ & 3338 & & & \\
\hline Between 4-5 & 2330 & 145 & $5.9 \%$ & 2475 & & & \\
\hline above 5 & 2483 & 178 & $6.7 \%$ & 2661 & & & \\
\hline \multicolumn{8}{|c|}{ Preceding birth interval } \\
\hline first birth & 2040 & 141 & $6.5 \%$ & 2181 & \multirow{4}{*}{75.291} & \multirow{4}{*}{3} & \multirow{4}{*}{$.000 * *$} \\
\hline below 24 & 1914 & 204 & $9.6 \%$ & 2118 & & & \\
\hline between $24-47$ & 4157 & 211 & $4.8 \%$ & 4368 & & & \\
\hline above 47 & 1895 & 79 & $4.0 \%$ & 1974 & & & \\
\hline \multicolumn{8}{|c|}{ Age of mother at first birth } \\
\hline below 20 & 6157 & 405 & $6.2 \%$ & 6562 & \multirow{3}{*}{2.528} & \multirow{3}{*}{2} & \multirow{3}{*}{.283} \\
\hline between $20-34$ & 3827 & 230 & $5.7 \%$ & 4057 & & & \\
\hline between $35-49$ & 22 & 0 & $0.0 \%$ & 22 & & & \\
\hline \multicolumn{8}{|l|}{ Household size } \\
\hline between 1-3 & 1156 & 143 & $11.0 \%$ & 1299 & \multirow{3}{*}{72.099} & \multirow{3}{*}{2} & \multirow{3}{*}{$.000 * *$} \\
\hline between 4-6 & 4982 & 304 & $5.8 \%$ & 5286 & & & \\
\hline above 6 & 3868 & 188 & $4.6 \%$ & 4056 & & & \\
\hline
\end{tabular}




\begin{tabular}{|c|c|c|c|c|c|c|c|}
\hline \multirow{2}{*}{ Variable } & \multicolumn{4}{|c|}{ Under-five child's mortality status } & \multirow{2}{*}{ Chi-square } & \multirow{2}{*}{ Df. } & \multirow{2}{*}{ p-value } \\
\hline & Alive & Dead & \% of U5CM & Total & & & \\
\hline \multicolumn{8}{|l|}{ Breastfeeding status } \\
\hline Ever breastfeeding, & 9622 & 444 & $4.4 \%$ & 10066 & \multirow{2}{*}{804.367} & \multirow{2}{*}{1} & \multirow{2}{*}{$.000 * *$} \\
\hline never breastfeed & 384 & 191 & $33.2 \%$ & 575 & & & \\
\hline \multicolumn{8}{|c|}{ Educational level of mother } \\
\hline no education & 6387 & 451 & $6.6 \%$ & 6838 & \multirow{4}{*}{20.699} & \multirow{4}{*}{3} & \multirow{4}{*}{$.000 * *$} \\
\hline primary education & 2538 & 140 & $5.2 \%$ & 2678 & & & \\
\hline Secondary & 697 & 37 & $5.0 \%$ & 734 & & & \\
\hline Higher & 384 & 7 & $1.8 \%$ & 391 & & & \\
\hline \multicolumn{8}{|c|}{ Wealth index of household } \\
\hline Poor & 5376 & 399 & $6.9 \%$ & 5775 & \multirow{4}{*}{21.332} & \multirow{4}{*}{2} & \multirow{3}{*}{$.000 * *$} \\
\hline Middle & 1386 & 80 & $5.5 \%$ & 1466 & & & \\
\hline Rich & 3244 & 156 & $4.6 \%$ & 3400 & & & \\
\hline \multicolumn{6}{|l|}{ Region } & & \\
\hline Tigray & 992 & 41 & $4.0 \%$ & 1033 & \multirow{12}{*}{34.474} & \multirow{11}{*}{10} & \multirow{11}{*}{$.000 * *$} \\
\hline Afar & 972 & 90 & $8.5 \%$ & 1062 & & & \\
\hline Amhara & 928 & 49 & $5.0 \%$ & 977 & & & \\
\hline Oromia & 1494 & 87 & $5.5 \%$ & 1581 & & & \\
\hline Somali & 1402 & 103 & $6.8 \%$ & 1505 & & & \\
\hline Benishangul & 815 & 64 & $7.3 \%$ & 879 & & & \\
\hline SNNPR & 1206 & 71 & $5.6 \%$ & 1277 & & & \\
\hline Gambela & 670 & 44 & $6.2 \%$ & 714 & & & \\
\hline Harari & 564 & 41 & $6.8 \%$ & 605 & & & \\
\hline Addis Ababa & 447 & 14 & $3.0 \%$ & 461 & & & \\
\hline Dire Dawa & 516 & 31 & $5.7 \%$ & 547 & & & \\
\hline \multicolumn{7}{|c|}{ Type of place of residence } & \\
\hline Urban & 1907 & 67 & $3.4 \%$ & 1974 & \multirow{2}{*}{28.602} & \multirow{2}{*}{1} & \multirow{2}{*}{$.000 * *$} \\
\hline Rural & 8099 & 568 & $6.6 \%$ & 8667 & & & \\
\hline \multicolumn{8}{|l|}{ Type of toilet facility } \\
\hline improved facility & 1729 & 61 & $3.4 \%$ & 1790 & & & \\
\hline unimproved facility & 3800 & 243 & $6.0 \%$ & 4043 & 28.116 & 2 & $.000 * *$ \\
\hline no toilet facility & 4477 & 331 & $6.9 \%$ & 4808 & & & \\
\hline Source of drinking $\mathrm{w}$ & & & & & & & \\
\hline protected source & 6011 & 338 & $5.3 \%$ & 6349 & & & \\
\hline unprotected source & 3995 & 297 & $6.9 \%$ & 4292 & 11.627 & 1 & $.001 * *$ \\
\hline Place of delivery & & & & & & & \\
\hline at home & 6781 & 490 & $6.7 \%$ & 7271 & 24360 & & \\
\hline at health facility & 3225 & 145 & $4.3 \%$ & 3370 & 24.360 & 1 & $.000^{* *}$ \\
\hline
\end{tabular}

*significant at $5 \%$ level, ** significant at $1 \%$ level.

Test of Association between dependent variable and its independent variables.

In order to find out whether each of the independent variables is associated with under-five child mortality, cross tabulations were done and chi-square tests of independence/association were performed.

The null hypothesis for testing the independence of two (categorical) variables is given by: $\mathrm{H}_{\mathrm{o}}$ the two (categorical) variables are independent (not associated). If $\mathrm{p}<0.05$, we reject the hypothesis of independence $\left(\mathrm{H}_{0}\right)$ at $5 \%$ of level of significance.

Using SPSS and STATA with tab or tabulate command, cross-tabulation analysis has been conducted. Based on the results displayed in Table 1, under-five child mortality was found to be associated with sex of a child $(\mathrm{p}=0.000)$, Age of child in month $(\mathrm{p}=0.000)$, Birth type $(\mathrm{p}=0.000)$, Birth order number $(\mathrm{p}=0.124)$, Preceding birth interval $(\mathrm{p}=0.000)$, household size $(p=0.000)$, breastfeeding status $(p=0.000)$, Educational level of mother's $(p=0.000)$, Wealth index of household $(\mathrm{p}=0.000)$, Region $(\mathrm{p}=0.000)$, Place of residence $(\mathrm{p}$ $=0.000)$, Type of toilet facility $(\mathrm{p}=0.000)$, Source of drinking water $(\mathrm{p}=0.001)$ and Place of delivery $(\mathrm{p}=0.000)$ at $25 \%$ level of significance. That is, the individual contribution of each of these predictors to under-five child mortality is significant. While, the covariate age of mother at first birth was not associated with under-five child mortality at $25 \%$ level of significance. Hence, all the significantly associated covariates are considered in the logistic regression model.

\subsection{Trends of Under-Five Child Mortality}

Before identifying the major determinates of under-five child mortality (U5CM) it is worthwhile to see the trends of U5CM. From 2000, 2005, 2011 to 2016 EDHS data found that all the under-five child mortality rate had declined over the last 16 years. The magnitude of decline varies among the components of under-five child mortality.

The 2016 EDHS showed that the neonatal mortality rate, the infant mortality rate and the under-five child mortality rate were 29, 48 and 67 death per 1000 live births respectively. In other word, in Ethiopia, 1 in every 35 children dies within in the first month, 1 in every 21 children dies before celebrating the first birthday and 1 of every 15 children dies before reaching the fifth birthday.

The neonatal mortality rate declined by $41 \%$ from 49 per 
1000 live births in the year 2000 to 29 per 1000 live births in 2016. Infant mortality also declined by $50 \%$ from 97 per 1000 live births in the year 2000 to 48 per 1000 live births in 2016 . Similarly under-five mortality has shown a continuous reduction from 166, 123, 88 deaths per 1000 live births in the years 2000, 2005, 2011 to 67 deaths per 1000 live births in 2016. This equates to a decline of 60 percent between the 2000 and the 2016 survey periods (Figure 1).

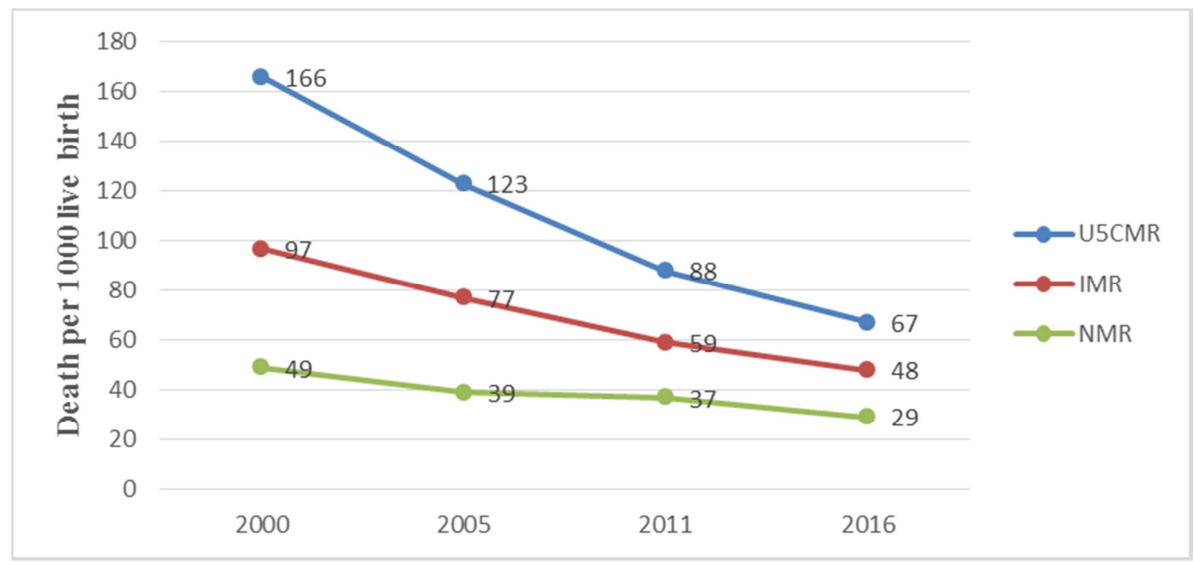

Figure 1. Trends in childhood mortality rate.

Figure 2 shows that U5CM for male children was consistently higher than that for female children between 2000 and 2016. Place of residence and regional variation are even more pronounced in the mortality of under-five children. Over the 16 years period between $2000 \& 2016$, the under-five child mortality in rural areas was consistently higher than that for urban areas (Figure 3).

Generally, the trend analysis U5CM showed that Ethiopia has achieved MDG4. However, U5CM is still higher than the under-five child mortality rate of other several low and middle income countries.

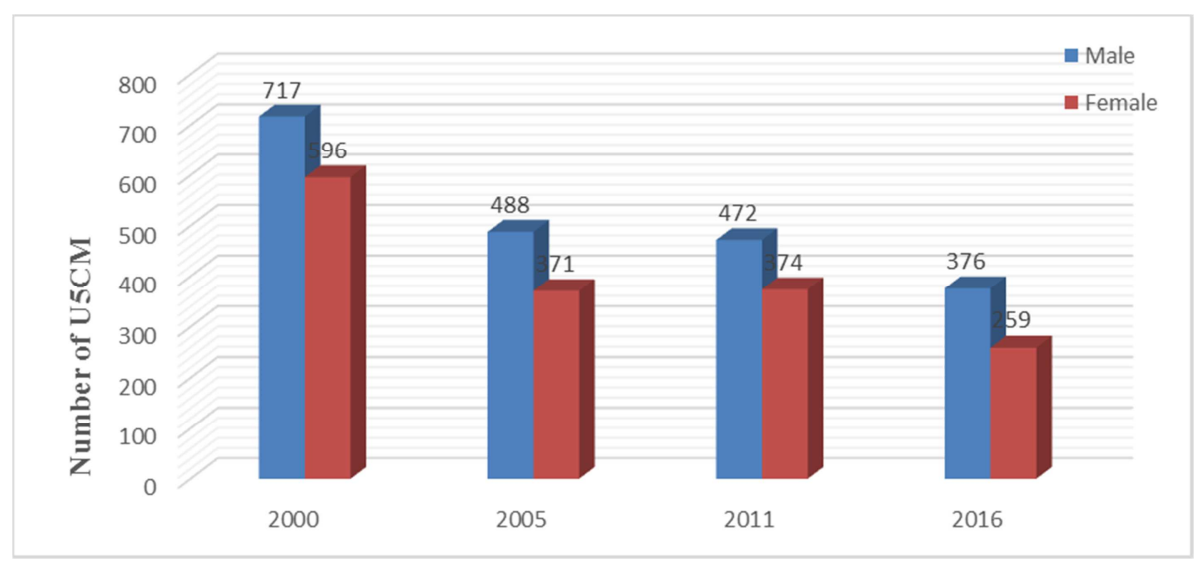

Figure 2. Trends of under-five child mortality by gender.

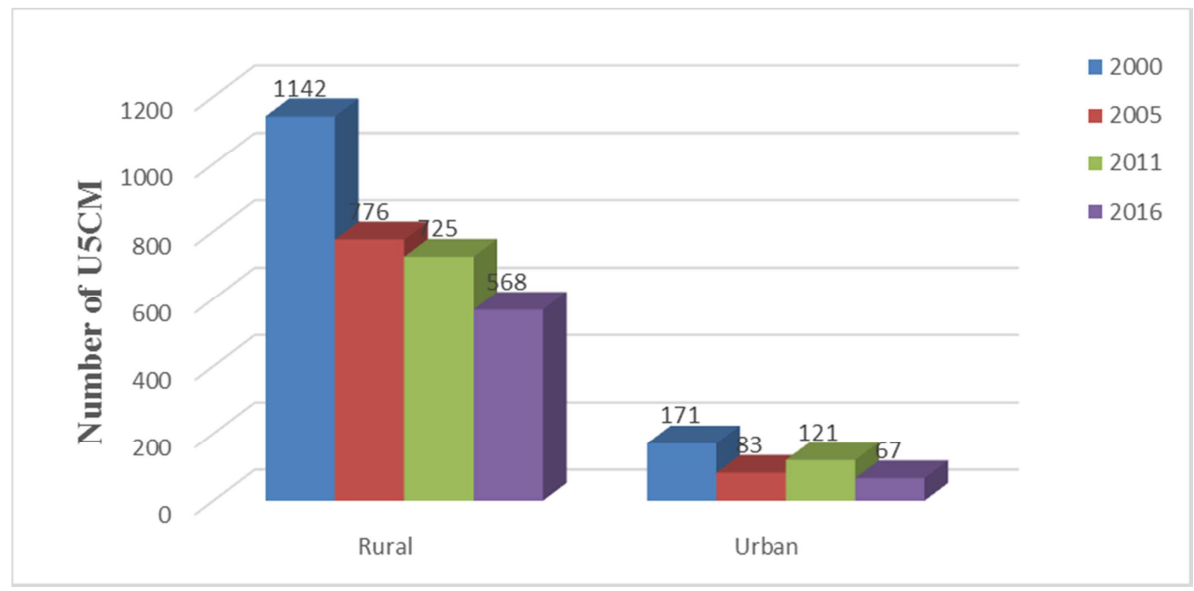

Figure 3. Trends of under-five child mortality by place of residence. 


\subsection{Results of Binary Logistic Regression Analysis}

In this section binary logistic regression was applied to assess the relationship between under-five mortality, which is a dichotomous response variable and the predictor variables.

Multiple binary logistic regression was used to analyze the effects of the explanatory variables on under-five child mortality. In fitting the binary logistic regression model, STATA was used. The statistical significance of the individual regression coefficients have been tested using the Wald chi-square statistic. Accordingly, Sex of the child, Age of the child in month, Birth type, Birth order number, Household Size, breastfeeding status, Region, Place of residence and Type of Toilet Facility were found to be significantly associated with under-five child mortality. Conversely, Preceding birth interval, Educational level of mothers', wealth index of Household, Source of drinking water and place of delivery were found insignificant at $5 \%$ significance level suggesting independence with under-five child mortality (See Table 4).

\subsubsection{Goodness of Fit of the Estimated Logistic Regression Model}

Before interpreting the results in Table 4, we have to check the goodness of fit of the model to the observed data. In order to check for the goodness-of-fit of an estimated multiple logistic regression model, one should assume that the model contains those variables that should be in the model and have been entered in the correct functional form.

The Likelihood Ratio Test

Likelihood ratio test is used to test the goodness of fit of the model by comparing two nested models:-one with small number of explanatory variables and the other with more explanatory variables. In our case, we compared two models; one with no variable called the empty (intercept only) model and the other with all variables included called the saturated (full) model. The null and alternative hypotheses to be tested are:

$\mathrm{H}_{0}$ : there is no significant difference between the empty model and the saturated model.

$\mathrm{H}_{\mathrm{A}}$ : not $\mathrm{H}_{0}$

Table 2. Overall Model Evaluation Using Likelihood Ratio Test (EDHS, 2016).

\begin{tabular}{|c|c|c|c|c|c|c|}
\hline Goodness of Fit measure & Log likelihood (LL) & Deviance $=-2 \mathrm{LL}$ & Chi-Sq $X^{2}{ }_{0.05}(D f)$ & Df. & AIC & BIC \\
\hline Null Model & -2405.631 & 4811.262 & 3.84 & 1 & 4813.262 & 4820.535 \\
\hline Full Model & -2009.34 & 4018.68 & 43.77 & 33 & 4084.68 & 4324.671 \\
\hline
\end{tabular}

Table 3. Hosmer-Lemeshow Goodness of Fit Statistics.

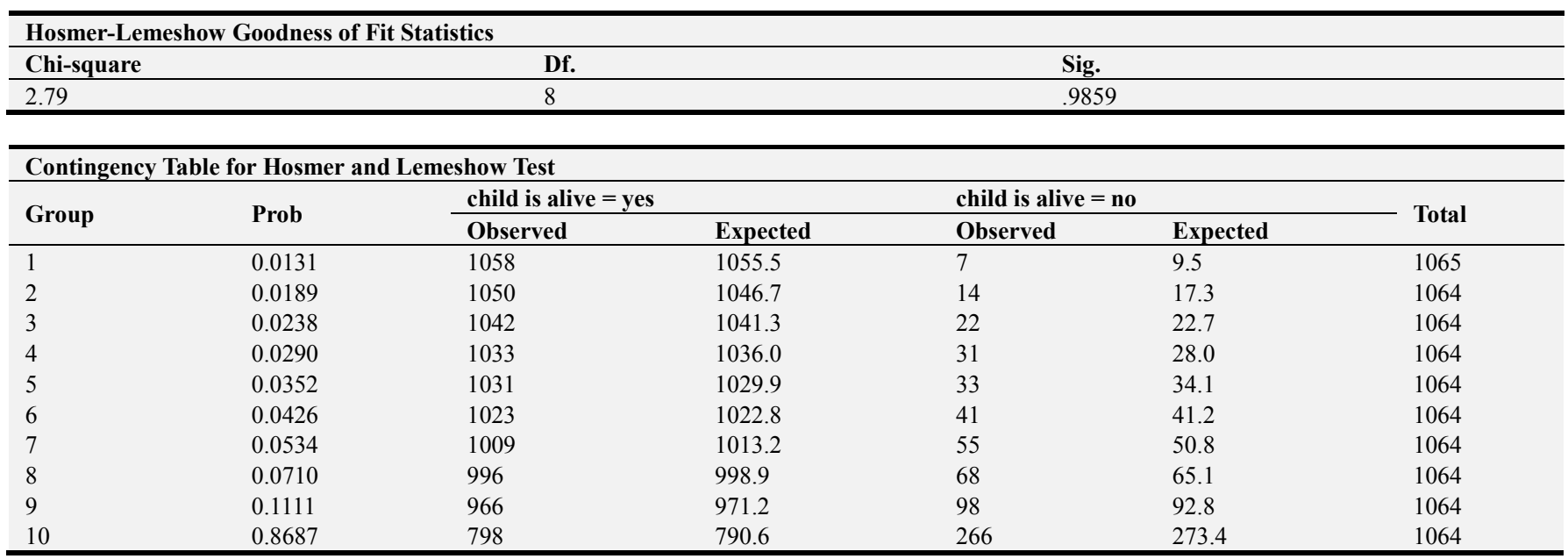

Number of observations $=10641$, Hosmer-Lemeshow chi2 $(8)=2.79$

Number of groups $=10$, Prob $>$ chi $2=0.9859$

The value for the likelihood ratio $G^{2}$ statistic is the difference between the $-2 \mathrm{LL}$ value of the empty model and the $-2 \mathrm{LL}$ value of the full model. Using the results in table 2, we can compute $G^{2}$ as the difference in $-2 \mathrm{LL}$ as $G^{2}=$ 4811.26-4018.68 $=792.58$. Since the likelihood ratio test statistics $G^{2}=792.58$ exceeds the tabulated value $\chi^{2}(33)$ $=43.773$ and $p$-value $=0.000$, we reject the null hypothesis of no significant difference between the two models and conclude that at least one of the predictors' was significantly related with under-five mortality.

To determine the more adequate model, we used AIC and $\mathrm{BIC}$ values. The smaller the AIC and BIC values the better the model. Table 2 showed that the full model is better than the empty model.

The Hosmer-Lemeshow Goodness of Fit Test

The Hosmer-Lemeshow goodness of fit test divides subjects into g classes (often declines) based on predicted probabilities and then computes a chi-square from observed and expected frequencies (usually in a $10 \times 2$ contingency table). A non-significant chi-square indicates that there is no difference between the observed and the model predicted values (classification) and hence estimates of the model adequately fit the data. The Hosmer-Lemeshow Goodness-of-fit test tests the hypotheses: 
Ho: the model adequately fits the data, vs

Ha: the model not adequately fits the data.

Since the $p$-value $=0.986>0.05$, we do not reject the null hypothesis of no difference between observed and model predicted values, implying that the estimated model adequately fits the data (see Table 3 ).

\subsubsection{Model Diagnostics: Influential Observations and Outliers}

So far, we have discussed some summary statistics and examined the goodness of fit of our model. Before concluding that the model is adequate, we have considered measures relevant for the detection of the presence of outliers and influential observations.
DFBETAs less than unity imply no specific impact of an observation on the estimates of the coefficient of a particular predictor variable, while Cook's distance less than unity indicate that an observation had no overall impact on the group estimates of regression coefficients $\beta$. Similarly, the value of the leverage statistic is less than one implying that no observation is far apart from the others in terms of the level of the covariates variables. So, the Maximum value of Cook's influence statistics and DFBETA for each indicator variables are less than 1, this indicated that there is no potential influential observation. Based on the above goodness of fit tests and diagnostic checking results, we can say that our model provide an adequate fit to the data.

Table 4. Binary Logistic Regression model fit results.

\begin{tabular}{|c|c|c|c|c|c|c|c|}
\hline \multirow{2}{*}{$\begin{array}{l}\text { Variables with categories } \\
\text { SEX OF CHILD (Male= ref.cat) }\end{array}$} & \multirow[t]{2}{*}{ Coef. } & \multirow[t]{2}{*}{ Std. Err. } & \multirow[t]{2}{*}{$\mathbf{z}$} & \multirow[t]{2}{*}{$\mathbf{P}>\mathbf{Z}$} & \multirow[t]{2}{*}{ aOR } & \multicolumn{2}{|c|}{$95 \%$ CI for OR } \\
\hline & & & & & & & \\
\hline Female & -0.283 & 0.089 & -3.16 & $0.002 * *$ & 0.754 & 0.633 & 0.898 \\
\hline Age of child in month & 0.013 & 0.003 & 4.69 & $0.000 * *$ & 1.013 & 1.007 & 1.018 \\
\hline \multicolumn{8}{|c|}{ BIRTH TYPE (Single birth=ref.cat) } \\
\hline multiple birth & 1.507 & 0.187 & 8.08 & $0.000 * *$ & 4.512 & 3.130 & 6.504 \\
\hline \multicolumn{8}{|c|}{ BIRTH ORDER NUMBER (First birth= ref.cat) } \\
\hline between $2-3$ & 0.709 & 0.664 & 1.07 & 0.286 & 2.031 & 0.552 & 7.472 \\
\hline above 5 & 1.367 & 0.683 & 2 & $0.045^{*}$ & 3.924 & 1.029 & 14.962 \\
\hline \multicolumn{8}{|c|}{ PRECEDING BIRTH INTERVAL (first birth= ref.cat) } \\
\hline below 24 & -0.129 & 0.667 & -0.19 & 0.847 & 0.879 & 0.238 & 3.247 \\
\hline between $24-47$ & -0.813 & 0.665 & -1.22 & 0.221 & 0.443 & 0.120 & 1.632 \\
\hline above 47 & -1.017 & 0.669 & -1.52 & 0.128 & 0.362 & 0.097 & 1.342 \\
\hline \multicolumn{8}{|c|}{ BREASTFEEDING STATUS (ever breastfeed= ref.cat) } \\
\hline never breastfeed & 2.402 & 0.111 & 21.68 & $0.000 * *$ & 11.050 & 8.892 & 13.731 \\
\hline \multicolumn{8}{|c|}{ NUMBERS OF HOUSEHOLD MEMBER (below 3= ref.cat) } \\
\hline above 6 & -1.767 & 0.166 & -10.66 & $0.000 * *$ & 0.171 & 0.123 . & 0.236 \\
\hline \multicolumn{8}{|c|}{ MOTHER EDUCATIONAL LEVEL (no educate= ref.cat) } \\
\hline Primary & -0.009 & 0.119 & -0.08 & 0.938 & 0.991 & 0.784 & 1.251 . \\
\hline Secondary & 0.019 & 0.220 & 0.09 & 0.93 & 1.020 & 0.663 & 1.568 \\
\hline Higher & -0.813 & 0.421 & -1.93 & 0.054 & 0.444 & 0.194 & 1.013 \\
\hline \multicolumn{8}{|c|}{ WEALTH INDEX OF HOUSEHOLD poor= ref.cat) } \\
\hline middle & -0.125 & 0.151 & -0.83 & 0.409 & 0.883 & 0.657 & 1.187 \\
\hline rich & 0.073 & 0.147 & 0.5 & 0.618 & 1.076 & 0.806 & 1.436 \\
\hline \multicolumn{8}{|l|}{ REGION (Tigray= ref.cat) } \\
\hline Afar & 0.542 & 0.214 & 2.53 & $0.011 *$ & 1.719 & 1.130 & 2.616 \\
\hline Amhara & 0.134 & 0.232 & 0.58 & 0.565 & 1.143 & 0.725 & 1.801 \\
\hline Oromia & 0.082 & 0.214 & 0.38 & 0.703 & 1.085 & 0.713 & 1.651 \\
\hline Somali & 0.209 & 0.212 & 0.98 & 0.325 & 1.232 & 0.813 & 1.868 \\
\hline Benishangul & 0.275 & 0.230 & 1.2 & 0.231 & 1.317 & 0.839 & 2.067 \\
\hline SNNPR & -0.109 & 0.223 & -0.49 & 0.625 & 0.897 & 0.580 & 1.387 \\
\hline Addis Ababa & 0.342 & 0.376 & 0.91 & 0.362 & 1.408 & 0.674 & 2.939 \\
\hline Dire Dawa & 0.558 & 0.265 & 2.11 & $0.035^{*}$ & 1.747 & 1.039 & 2.937 \\
\hline \multicolumn{8}{|c|}{ PLACE OF RESCIDENCE (Urban= ref.cat) } \\
\hline Rural & 0.526 & 0.196 & 2.68 & $0.007 * *$ & 1.692 & 1.152 & 2.484 \\
\hline \multicolumn{8}{|c|}{ TYPE OF TOILET FACILITY (improved facility= ref.cat) } \\
\hline unimproved facility & 0.556 & 0.181 & 3.08 & $0.002 * *$ & 1.743 & 1.223 & 2.484 \\
\hline no toilet facility & 0.442 & 0.185 & 2.39 & $0.017 *$ & 1.556 & 1.083 & 2.236 \\
\hline \multicolumn{8}{|c|}{ SOURCE OF DRINKING WATER (protected source= ref.cat) } \\
\hline unprotected source & 0.133 & 0.097 & 1.37 & 0.17 & 1.142 & 0.945 & 1.382 \\
\hline \multicolumn{8}{|c|}{ PLACE OF DELIVERY (at home= ref.cat) } \\
\hline at health facility & -0.121 & 0.128 & -0.95 & 0.343 & 0.886 & 0.690 & 1.138 \\
\hline cons & -3.704 & 0.341 & -10.87 & $0.000 *$ & 0.025 & 0.013 & 0.048 \\
\hline
\end{tabular}

*Significant at $5 \%$ level. ** Significant at $1 \%$ level, Ref.cat= reference category. 


\subsubsection{Interpretation of Binary Logistic Regression Model Fit Results}

The statistical significance of individual regression coefficients is tested using the Wald chi-square statistic. The results in Table 4 revealed that Sex of the child, Age of the child in month, Birth type, Birth order number, Number of Household Size, Duration of breastfeeding status, Region, Place of residence and type of toilet facility had significant effects on under-five child mortality at 5\% level of significance. Conversely, preceding birth interval, Educational level of mother's, wealth index Household, Source of drinking water and place of delivery were found insignificant. The test of significance of the intercept also indicated that it is significant implying that it should be included in the model. For all explanatory variables, the first category was taken as the reference category.

A more appealing way to interpret the regression coefficients in logistic model is using odds ratios. The odds ratio indicates the effect of each explanatory variable directly on the odds of dying (exposure) rather than on the odds of survival (control, unexposed or reference group) of under- five children. Estimates of odds ratio greater than 1.0 indicate that the risk of having under-five child mortality is greater than that for the reference category. Estimates less than 1.0 indicate that the risk of death of an under- five child is less than that for the reference category of each variable while an estimated odds ratio of one indicate no significant difference in the risk of death between any category and the reference category. So, the standard logistic regression model presented in Table 4 is interpreted in terms of odds ratios as follows.

Female children had reduced risks of dying before 5 years of age compared to male children $(\mathrm{aOR}=0.754, \mathrm{p}=0.002)$. Female children were about $25 \%(\mathrm{aOR}=0.754, \mathrm{p}=0.002)$ less likely to die before 5 years of age compared to male children controlling for other variables in the model.

In this study, child's agehad a statistically significant effect on under-five child mortality. For a one month increase in age, the odds of having mortality increased by $1.3 \%(\mathrm{OR}=1.013)$. The odds of death of under-five children among multiple births was $4.512(\mathrm{aOR}=4.512)$ times higher than the odds of death of under-five among single births.

Breastfeeding status showed a statistically significant effect on under-five child mortality. Children that were never breastfed had increased risk of dying before five years of age compared to children who were ever breastfed $(\mathrm{aOR}=11.05)$.

Children in households of size 4-6 were $67.3 \%(\mathrm{aOR}=0.327)$ less likely to die before the age of 5 compared to children in households of size two or less (below three). Similarly, children in households of size 6 or more were $82.9 \%$ $(\mathrm{aOR}=0.171)$ less likely to die before the age of 5 compared to children in households of size two or less. These results are contrary to our expectations.

When we look at regional effects on under-five child mortality, under-five children in Afar were about $72 \%$ more likely to die ( $\mathrm{aOR}=1.719)$, those in Harari region were about $84 \%$ more likely to die $(\mathrm{aOR}=1.839)$ and those in Dire Dawa region were about $75 \%$ more likely to die $(\mathrm{aOR}=1.747)$ compared to under-five children in Tigray region.

Likewise, under-five children in Rural areas were about $69 \%(\mathrm{aOR}=1.692)$ more likely to die compared to those in Urban areas.

Finally, The odds of under-five child mortality among children in households using unimproved toilet facility were 1.74 times the odds of under-five child mortality among children in households having improved toilet facilities and children in households with no toilet facilities were 1.56 times more likely to die compared to under-five children in households with improved toilet facilities, controlling for the other variables in the model.

\subsection{Results of Multilevel Logistic Regression Analysis}

In this study, a two-level structure is used with children at level-1 nested within region at level-2 in order to see the existence of variation with regard to child mortality within and between regions of Ethiopia. In the 2016 EDHS, the 11 regions are considered as level-2 with a total of 10641 children considered as level-1. The data used in this study consist of variables describing individuals as well as groups (regions).

We have considered three multilevel logistic regression models: the empty model, random intercept with fixed effects model and the random coefficient with random intercept model. We have also presented results of model comparison, goodness of fit test and provided interpretations of the fixed effects in terms of odds ratios.

Test of Heterogeneity

Before attempting to multilevel analysis, as one of the aims of this study, we have to test for the heterogeneity of under-five child mortality among regional states of Ethiopia. The chi-square test was applied to assess heterogeneity between regions. As shown in Table 1, the Pearson chi-square value is, $\chi^{2}(10)=34.474$ with $\mathrm{P}$-value $=0.000$, implying that there is strong evidence of heterogeneity of under-five child mortality across regional states of Ethiopia.

\subsubsection{Results of the Empty Model with Random Intercept}

The empty two-level model also called the null two-level model for a dichotomous outcome variable refers to a population of groups (level-two units i.e. regions) and specifies the probability distribution for group-dependent probabilities, $\pi_{j}$. It is the model that incorporates only the grand mean and random intercept (regional effect) without any covariate.

It is given by: $-\operatorname{logit}\left(\pi_{\mathrm{j}}\right)=\beta_{0}+U_{0 j}$, where $U_{0 j} \sim \operatorname{IID}\left(0, \sigma_{0}^{2}\right)$.

The intercept $\beta_{0}$ also known as the grand (population) mean is shared by all regions while the random effect $U_{0 j}$ also known as level two residual is specific to region $\mathrm{j}$. It shows how the mean of under-five children mortality in a particular region deviates from the grand mean. $\sigma_{0}^{2}$ is the between regions variance.

As shown in Table 2 and 9, the deviance based chi-square $10.56=(4811.262-4800.7)$ is greater than $\chi^{2}=3.84$ at $1 \mathrm{df}$ and $\mathrm{p}$-value $=0.0006$. This result indicates that the empty model 
with random intercept is more appropriate than the empty model without random intercept in predicting under-five child mortality in Ethiopia.

As the results in Table 5 show, the estimate of the fixed part of the model is -2.788 which is the log odds of under-five child mortality across all regions. The fixed part of the model is interpreted as the grand mean of the log odds of under-five child mortality with odds of $\exp (-2.788)=0.062$ which is the same as the sample ratio of 635 deaths to 10006 alive. The average probability of under-five child mortality is $\frac{\exp ^{(-2.788)}}{1+\exp (-2.788)}=0.058$ which means that the chance of under-five child mortality is $5.8 \%$ on average. The table also contains the variance estimate of the random effects at regional level, $\sigma_{0}^{2}=0.044$ and we can calculate intra-class correlation coefficient which is the measure of the correlation between two individuals who are in the same higher level unit (region). A low ICC indicates relatively small between region variations. In other words, regions tend to perform at comparable levels to reduce under-five child mortality. As ICC increases, then regions perform with ever increasing variations to reduce under-five child mortality. The between regions variance is 0.044 whilethe level one variance is $\pi^{2} / 3$
=3.29. From equation $I C C=\frac{\sigma_{0}^{2}}{\sigma_{0}^{2}+3.29}$ the intra-region correlation has been computed to be 0.013 which is very small. This result implies that $1.3 \%$ of the variation in the under-five child mortality can be explained by grouping the children in regions. The remaining $(100-1.3 \%=98.7 \%)$ of the variation in the under-five child mortality is explained within region-lower level units.

The random effect tests examine whether or not the random intercept (between-region) variance is needed for these data. The likelihood ratio statistics for testing the null hypothesis,

Ho: $\sigma_{0}^{2}=0$ which means there is no cross-regional variation in U5CM in Ethiopia.

$\mathrm{H}_{1}: \sigma_{0}^{2}>0$. For this hypothesis, the variance component test lies on the boundary of the parameter space, the likelihood ratio test can break down asymptotically. At the bottom of the table, the value of the test statistics and the corresponding $\mathrm{p}$-value for testing the hypothesis $\mathrm{H}_{0}: \sigma_{0}^{2}=0$ that there is no cross-regional variation in under-five mortality are presented. Since the value of the test statistic is 10.56 with $p=0.0006$, the null hypothesis is rejected and we conclude that there is strong evidence of heterogeneity or cross-regional variation in under-five mortality incidence in Ethiopia.

Table 5. Results for Multi-level Logistic Regression Model without covariate.

\begin{tabular}{|c|c|c|c|c|c|c|}
\hline Fixed-effect & Coef. & Std. Err. & $\mathbf{Z}$ & $\mathbf{P}>\mathbf{Z}$ & \multicolumn{2}{|c|}{ [95\% Conf. Interval] } \\
\hline cons & -2.788 & 0.077 & -35.98 & 0.000 & -2.940 & -2.636 \\
\hline $\begin{array}{l}\bar{R} \text { Random-effects parameters } \\
\text { REGION: Identity }\end{array}$ & Estimate & Std. Err. & \multicolumn{4}{|c|}{ [95\% Conf. Interval] } \\
\hline $\operatorname{Var}(\mathrm{Uoj})=\sigma_{0}^{2}$ & 0.044 & 0.029 & 0.012 & & 0.162 & \\
\hline Rho $(\rho)=\mathrm{ICC}$ & 0.013 & & & & & \\
\hline
\end{tabular}

LR test vs. logistic regression: chibar2 $(01)=10.56$ Prob $>=$ chibar2 $=0.0006$, log likelihood $=-2400.35$, deviance $=4800.7$, AIC $=4804.7$ and $\mathrm{BIC}=4819.245$ with $2 \mathrm{df}$.

We can now write the model for the $\mathrm{j}^{\text {th }}$ region as $\operatorname{logit}\left(\pi_{\mathrm{j}}\right)$ $=-2.788+\mathrm{U}_{0 \mathrm{j}}$. Based on the estimated model, we can say that the average probability of under-five child mortality in the absence of covariates in region $j$ is less than the average when $U_{0 j}$ is negative while it is higher than the average when $U_{0 j}$ is positive.
Table 6 contains the estimated values of $U_{0 j}$. The results indicate that the probability of under-five child mortality is less than the average in Addis Ababa, Tigray, Amhara, Oromia, SNNPR and Dire Dawa while it is higher than the average for the remaining regions. The worst situation has been observed in Affar and Benishangul-Gumu.

Table 6. The Estimate of Random Effect for each Region.

\begin{tabular}{llll}
\hline Rank & Region & Uoj & Standard error Uoj \\
\hline 1 & Addis Ababa & -0.2870136 & 0.0441555 \\
2 & Tigray & -0.2623423 & 0.0452085 \\
3 & Amhara & -0.1078248 & 0.052367 \\
4 & Oromia & -0.0467102 & 0.0554841 \\
5 & SNNPR & -0.0368982 & 0.0560006 \\
6 & Dire Dawa & -0.0183893 & 0.0569871 \\
7 & Gamebela & 0.03371038 & 0.0600437 \\
8 & Harari & 0.0985048 & 0.0636041 \\
9 & Somali & 0.139467 & 0.0660878 \\
10 & Benishangul & 0.1704585 & 0.0680265 \\
11 & Afar & 0.3136441 & 0.0776853 \\
\hline
\end{tabular}

The Caterpillar Plot with the Region Effects with 95\% Confidence Intervals.

The caterpillar plot (Figure 4), with the region effects shown in rank order (together with 95\% confidence interval) shows the estimated region effects or residuals for all 11 regions in the sample obtained from the null model. For the majority of the regions, the $95 \%$ confidence interval does not overlap the horizontal line at zero, indicating that uptake of under-five child mortality in these regions was significantly above average (above the zero line) or below average (below the zero line). This indicates that the intercept of under-five child mortality varied across the regions. 


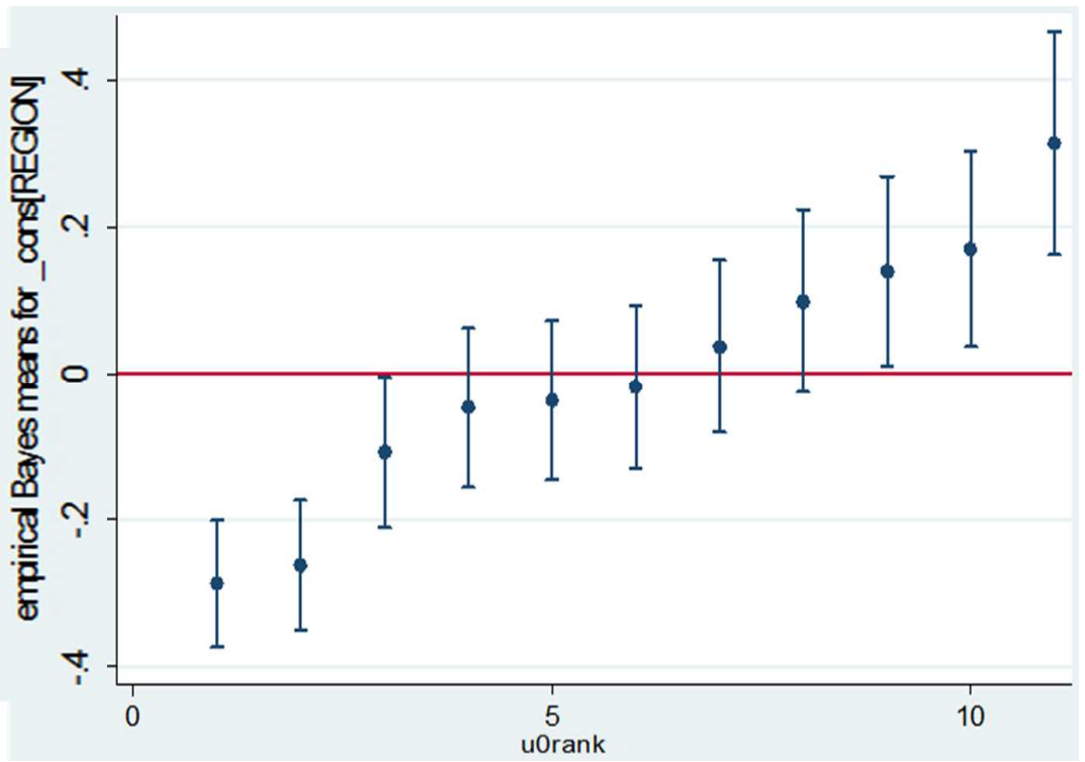

Figure 4. Caterpillar plot with the region effects.

Table 7. Estimates for the random intercept with fixed coefficient model.

\begin{tabular}{|c|c|c|c|c|c|c|c|c|}
\hline \multirow{2}{*}{$\begin{array}{l}\text { COVARIATES } \\
\text { SEX OF CHILD (Male = ref.cat }\end{array}$} & \multirow[t]{2}{*}{ Coef. } & \multirow[t]{2}{*}{ Std.Err. } & \multirow[t]{2}{*}{ Z-value } & \multirow{2}{*}{\multicolumn{2}{|c|}{$\mathbf{p}>\mathbf{z}$}} & \multirow[t]{2}{*}{ aOR } & \multicolumn{2}{|c|}{ 95\% Conf. Interval for OR } \\
\hline & & & & & & & & \\
\hline Female & -0.282 & 0.089 & -3.17 & $0.002 * *$ & & 0.754 & 0.633 & 0.898 \\
\hline AGEOF CHILDS (M) & 0.012 & 0.003 & 4.61 & $0.000^{* *}$ & & 1.013 & 1.007 & 1.018 \\
\hline \multicolumn{9}{|c|}{ BIRTH TYPE (Single birth=ref.cat) } \\
\hline Multiple birth & 1.508 & 0.186 & 8.1 & $0.000^{* *}$ & & 4.519 & 3.138 & 6.508 \\
\hline \multicolumn{9}{|c|}{ BIRTH ORDER NUMBER (First birth= ref.cat) } \\
\hline between 2-3 & 0.738 & 0.670 & 1.1 & 0.27 & & 2.091 & 0.563 & 7.763 \\
\hline Between 4-5 & 1.104 & 0.683 & 1.62 & 0.106 & & 3.0175 & 0.791 & 11.511 \\
\hline above 5 & 1.379 & 0.687 & 2.01 & $0.045^{*}$ & & 3.971 & 1.032 & 15.276 \\
\hline \multicolumn{9}{|c|}{ PRECEDING BIRTH INTERVAL (first birth= ref.cat) } \\
\hline below 24 & -0.149 & 0.671 & -0.22 & 0.824 & & 0.862 & 0.231 & 3.211 \\
\hline between $24-47$ & -0.852 & 0.669 & -1.27 & 0.203 & & 0.427 & 0.115 & 1.585 \\
\hline above 47 & -1.071 & 0.673 & -1.59 & 0.112 & & 0.343 & 0.0923 & 1.282 \\
\hline \multicolumn{9}{|c|}{ BREASTFEEDING STATUS (ever breastfeed= ref.cat) } \\
\hline never breastfeed & 2.382 & 0.110 & 21.61 & $0.000^{* *}$ & & 10.828 & 8.724 & 13.440 \\
\hline \multicolumn{9}{|c|}{ HOUSEHOLD SIZE (below $3=$ ref.cat) } \\
\hline between $4-6$ & -1.118 & 0.133 & -8.41 & $0.000^{* *}$ & & 0.327 & 0.252 & 0.424 \\
\hline above 6 & -1.762 & 0.165 & -10.65 & $0.000^{* *}$ & & 0.172 & 0.124 & 0.238 \\
\hline \multicolumn{9}{|c|}{ MOTHER EDUCATIONAL LEVEL (no educate= ref.cat) } \\
\hline Primary & -0.033 & 0.118 & -0.28 & 0.78 & & 0.968 & 0.768 & 1.219 \\
\hline Secondary & -0.007 & 0.216 & -0.03 & 0.976 & & 0.993 & 0.651 & 1.516 \\
\hline Higher & -0.845 & 0.420 & -2.01 & $0.044 *$ & & 0.429 & 0.189 & 0.978 \\
\hline \multicolumn{9}{|c|}{ WEALTH INDEX OF HOUSEHOLD poor= ref.cat) } \\
\hline Middle & -0.159 & 0.149 & -1.07 & 0.287 & & 0.853 & 0.636 & 1.143 \\
\hline Rich & 0.052 & 0.144 & 0.36 & 0.716 & & 1.054 & 0.794 & 1.398 \\
\hline \multicolumn{9}{|c|}{ PLACE OF RESCIDENCE (Urban= ref.cat) } \\
\hline Rural & 0.453 & 0.180 & 2.39 & $0.017 *$ & & 1.573 & 1.085 & 2.281 \\
\hline \multicolumn{9}{|c|}{ TYPE OF TOILET FACILITY (improved facility= ref.cat) } \\
\hline unimproved facility & 0.502 & 0.176 & 2.85 & $0.004^{* *}$ & & 1.652 & 1.170 & 2.334 \\
\hline no toilet facility & 0.425 & 0.181 & 2.35 & $0.019 *$ & & 1.530 & 1.073 & 2.182 \\
\hline \multicolumn{9}{|c|}{ SOURCE OF DRINKING WATER (protected source= ref.cat) } \\
\hline unprotected source & 0.127135 & 0.095778 & 1.33 & 0.184 & & 1.13557 & 0.9412132 & 1.370 \\
\hline \multicolumn{9}{|c|}{ PLACE OF DELIVERY (at home= ref.cat) } \\
\hline at health facility & -0.122 & 0.125 & -0.97 & 0.331 & & 0.885 & 0.693 & 1.132 \\
\hline cons & -3.334 & 0.281 & -11.85 & $0.000^{* *}$ & & 0.036 & 0.021 & 0.062 \\
\hline Random-effects Parameters & Estimate & Std. Err. & \multicolumn{6}{|c|}{ [95\% Conf. Interval] } \\
\hline REGION: Identity Var (_cons) & 0.023 & 0.020 & 0.063 & & 0.361 & & & \\
\hline
\end{tabular}

*significant at $5 \%$ level,

LR test vs. logistic model: $\operatorname{chibar} 2(01)=3.21$ Prob $>=$ chibar $2=0.0367 \log$ likelihood $=-2018.79$, deviance $=4037.58$, AIC $=4085.58$ and $\mathrm{BIC}=4260.12$ with 24 df. 


\subsubsection{Results of Random Intercept with Fixed Coefficient Model}

In a random intercept with fixed coefficient model, we allow the probability of under-five child mortality to vary across regions assuming that the effects of the explanatory variables are the same for each region. That is, the random intercept varies across regions, but children level covariates variables are considered fixed across regions. The results of the two-level random intercept with fixed coefficient model obtained using STATA are presented in the following tables.

The results for the fixed part of the random intercept with fixed coefficient model show that the sex of child, age of child, birth type, birth order number (above five), breast feeding, household size, mothers' educational level (higher), place of residence and type of toilet facility are significant determinants of variation in under-five child mortality of all regions with respect to the corresponding reference categories (see Table 7). The estimated coefficients and odds ratios have similar interpretation as in binary logistic regression discussed above. However, the result for the random part has additional information which is discussed below.

The results in Table 5 and Table 7 show that, the variance component representing variation between regions has decreased from 0.044 in the empty model with random intercept to 0.023 in the random intercept with fixed coefficients multilevel logistic regression model. The reduction of the random effect of the intercept variation is due to the inclusion of fixed explanatory variables. That is, taking into account the fixed independent variables can provide extra prediction values on under-five child mortality in each region.

The deviance-based chi-square, $G^{2}=18.9$ is the difference in deviance between the full model for the single level logistic regression model (deviance=4018.68) and the random intercept with fixed coefficient model (deviance=4037.58). The likelihood ratio test of the null hypothesis that there is no significant difference between the two models gives $G^{2}=18.9$. This value is compared to a chi-square distribution with 9 degrees of freedom. The tabulated value was $\chi^{2}(9)=16.91$ and, $G^{2}=18.9>\chi^{2}(9)=16.91$, with $\mathrm{p}=0.000<0.05$. This implies that the null hypothesis should be rejected which indicates that the random intercept with fixed coefficients model is a better fit as compared to the empty model with random intercept. In addition, the $\mathrm{AIC}$ and $\mathrm{BIC}$ values for the random intercept with fixed effect model (AIC=4085.58, $\mathrm{BIC}=4260.12)$ are less than that of the intercept-only model with random effect $(\mathrm{AIC}=4804.7, \mathrm{BIC}=4819.245)$. These results indicate that the random intercept with fixed coefficient model gives a better fit to the data than the empty model with random intercept for predicting under-five child mortality among regions in Ethiopia (see Table 9). In addition to this, the test of the null hypothesis Ho: $\sigma_{0}^{2}=0$ that there is no cross-regional variation in under-five children mortality in Ethiopia is rejected because the likelihood ratio test versus logistic regression resulted in chibar2 $(01)=18.9$ Prob $>$ chibar2 $=$ 0.000 implying evidence of heterogeneity or cross-regional variation in mortality of under- five children for the random intercept with fixed effects model. We can, therefore, conclude that the random effect at regional level is significantly different from zero.

\subsubsection{Results of Random Intercept with Random Coefficient Model}

Multilevel logistic regression analysis allows the coefficient of level-one independent variables to vary across regions instead of keeping them fixed across the regions. This allows region to have different coefficient, implying that the coefficient of independent variables are random at level two (region-level). The effect of breastfeeding has been examined by allowing it to vary randomly across regions. We investigated whether level-one independent variables have random effects across regions or the same effects across regions.

Estimates of this model show that the variance of the random coefficient of all included variables is zero except that for breastfeeding. This indicate that only the effect of breastfeeding on under-five child mortality varied across regions whereas the effect of the other independent variables on under-five child mortality remain fixed across regions. Results of the random coefficient estimates are presented in Table 8 below.

Table 8. Results of random intercept with random coefficient model.

\begin{tabular}{|c|c|c|c|c|c|c|c|}
\hline \multirow{2}{*}{$\begin{array}{l}\text { COVARIATES } \\
\text { SEX OF CHILD (Male= ref.cat) }\end{array}$} & \multirow[t]{2}{*}{ Coeff. } & \multirow[t]{2}{*}{ Std.Err. } & \multirow[t]{2}{*}{$\mathbf{Z}$} & \multirow[t]{2}{*}{$\mathbf{p}>\mathbf{z}$} & \multirow[t]{2}{*}{ aOR } & \multicolumn{2}{|c|}{ 95\% Conf. interval for $\mathrm{OR}$} \\
\hline & & & & & & & \\
\hline Female & -0.268 & 0.090 & -2.98 & $0.003 * *$ & 0.765 & 0.641 & 0.912 \\
\hline AGEOF CHILDS (M) & 0.013 & 0.003 & 4.77 & $0.000 * *$ & 1.013 & 1.008 & 1.019 \\
\hline Multiple birth & 1.520 & 0.189 & 8.06 & $0.000 * *$ & 4.574 & 3.161 & 6.620 \\
\hline \multicolumn{8}{|c|}{ BIRTH ORDER NUMBER (First birth= ref.cat) } \\
\hline between $2-3$ & 0.817 & 0.675 & 1.21 & 0.226 & 2.265 & 0.603 & 8.499 \\
\hline Between 4-5 & 1.193 & 0.689 & 1.73 & 0.084 & 3.296 & 0.854 & 12.721 \\
\hline above 5 & 1.458 & 0.693 & 2.1 & $0.035^{*}$ & 4.299 & 1.105 & 16.726 \\
\hline \multicolumn{8}{|c|}{ PRECEDING BIRTH INTERVAL (first birth= ref.cat) } \\
\hline below 24 & -0.254 & 0.677 & -0.37 & 0.708 & 0.776 & 0.206 & 2.925 \\
\hline between $24-47$ & -0.920 & 0.675 & -1.36 & 0.173 & 0.399 & 0.106 & 1.496 \\
\hline above 47 & -1.193 & 0.679 & -1.76 & 0.079 & 0.303 & 0.080 & 1.148 \\
\hline \multicolumn{8}{|c|}{ BREASTFEEDING (ever breastfeed= ref.cat) } \\
\hline never breastfeed & 2.584 & 0.324 & 7.98 & $0.000 * *$ & 13.253 & 7.025 & 25.002 \\
\hline \multicolumn{8}{|c|}{ HOUSEHOLD SIZE (below $3=$ ref.cat) } \\
\hline between $4-6$ & -1.140 & 0.134 & -8.49 & $0.000 * *$ & 0.320 & 0.246 & 0.416 \\
\hline
\end{tabular}




\begin{tabular}{|c|c|c|c|c|c|c|c|}
\hline \multirow{2}{*}{$\begin{array}{l}\text { COVARIATES } \\
\text { MOTHER EDUCATION }\end{array}$} & \multirow{2}{*}{$\frac{\text { Coeff. }}{\text { (no educ }}$} & \multirow{2}{*}{$\frac{\text { Std.Err. }}{\text { cat) }}$} & \multirow[t]{2}{*}{$\mathbf{Z}$} & \multirow[t]{2}{*}{$\mathbf{p}>\mathbf{z}$} & \multirow[t]{2}{*}{ aOR } & \multicolumn{2}{|c|}{$95 \%$ Conf. interval for $\mathrm{OR}$} \\
\hline & & & & & & & \\
\hline Primary & -0.039 & 0.118 & -0.33 & 0.744 & 0.962 & 0.763 & 1.213 \\
\hline Secondary & -0.020 & 0.217 & -0.09 & 0.926 & 0.980 & 0.640 & 1.501 \\
\hline Higher & -0.875 & 0.432 & -2.02 & $0.043^{*}$ & 0.417 & 0.176 & 0.973 \\
\hline \multicolumn{8}{|c|}{ WEALTH INDEX OF HOUSEHOLD poor= ref.cat) } \\
\hline Middle & -0.127 & 0.151 & -0.84 & 0.402 & 0.881 & 0.655 & 1.185 \\
\hline Rich & 0.078 & 0.145 & 0.54 & 0.588 & 1.081 & 0.815 & 1.436 \\
\hline \multicolumn{8}{|c|}{ PLACE OF RESCIDENCE (Urban= ref.cat) } \\
\hline Rural & 0.498 & 0.195 & 2.56 & $0.011 *$ & 1.645 & 1.123 & 2.408 \\
\hline \multicolumn{8}{|c|}{ TYPE OF TOILET FACILITY (improved facility $=$ ref.cat) } \\
\hline unimproved facility & 0.477 & 0.179 & 2.67 & $0.008 * *$ & 1.611 & 1.135 & 2.286 \\
\hline no toilet facility & 0.444 & 0.184 & 2.41 & $0.016^{*}$ & 1.559 & 1.087 & 2.237 \\
\hline \multicolumn{8}{|c|}{ SOURCE OF DRINKING WATER (protected source $=$ ref.cat) } \\
\hline unprotected source & 0.117 & 0.096 & 1.22 & 0.221 & 1.125 & 0.932 & 1.357 \\
\hline \multicolumn{8}{|c|}{ PLACE OF DELIVERY (at home= ref.cat) } \\
\hline at health facility & -0.151 & 0.128 & -1.18 & 0.237 & 0.860 & 0.669 & 1.105 \\
\hline _cons & -3.428 & 0.289 & -11.86 & $0.000 * *$ & 0.032 & 0.018 & 0.057 \\
\hline \multicolumn{8}{|l|}{$\overline{\mathrm{R}} \mathrm{EGION}$} \\
\hline $\operatorname{var}(\mathrm{BFEED})=\operatorname{Var}\left(U_{6 j}\right)$ & 0.967 & 0.495 & & & 0.967 & 0.354 & 2.639 \\
\hline $\operatorname{var}($ cons $)=\operatorname{Var}\left(U_{0 j}\right)$ & 1.451 & 0.743 & & & 1.451 & 0.532 & 3.960 \\
\hline $\left.\operatorname{Cov}\left(U_{0 j}, U_{6 j}\right)\right)=\sigma_{06}$ & -1.182 & 0.604 & -1.96 & 0.05 & -1.182 & -2.366 & 0.002 \\
\hline
\end{tabular}

LR test vs. logistic regression: $\operatorname{chi} 2(3)=46.02$ Prob $>$ chi2 $=0.0000$ Log likelihood=-1997.39, deviance=3994.78, AIC=4046.77 and BIC $=4235.86$ with 26 df.

In Table 8 , the values of var $\left(U_{0 j}\right)$ and var $\left(U_{6 j}\right)$ are the estimated variance of the intercept and the coefficient of breastfeeding respectively. These estimated variances are significant which suggest that the intercept and coefficient of breastfeeding status vary significantly. So, there is a significant variation in the effect of breastfeeding across regions of Ethiopia.

The effect of the intercept on region $\mathrm{j}$ is estimated to be $-3.428+U_{0 j}$. The intercept variance of 1.45 with standard error 0.7 is interpreted as the between-region variance when all other variables are held constant (i.e. equal to zero). Their mean is -3.428 with standard error 0.289 . The between region variance for the coefficient of breastfeeding is estimated to be 0.967 with standard error is 0.495 . The negative covariance estimate of -1.182 with standard error 0.604 between intercept and coefficient of breastfeeding suggest that regions with a higher intercept than average tend to have a flatter-than average coefficient.

Generally, interpretation of significant covariance terms can be easily made in terms of the correlation coefficient between the random intercept and the random coefficient. Positive covariance/correlation between the random intercept and the random coefficient implies that regions with higher intercepts tend to have on average higher coefficient on the corresponding predictors. The negative sign for the correlation between the random intercepts and coefficients imply that regions with higher intercepts tend to have on average lower coefficients on the corresponding predictors. The intercept-coefficient correlation between intercept and coefficient of breastfeeding is estimated as follows.

$$
\begin{gathered}
\rho_{02}=\frac{-1.182}{\sqrt{0.967 * 1.451}}=-0.998 \text { (correlation between intercept and } \\
\text { coefficient of breastfeeding). }
\end{gathered}
$$

The random coefficient logistic regression model involves two extra parameters: the variance of the coefficient residuals (i.e. breastfeeding status), $U_{6 j}$ and their covariance with the intercept residuals $\sigma_{06}$. The change (which is also the change in deviance) can be regarded as a $\chi^{2}$ value with 1 degree of freedom. Under the null hypothesis that the extra parameters have population value of zero, the value of the deviance based chi-square is given by $(4037.58-3994.78=42.8, \mathrm{p}$-value $=$ 0.000 ) which shows that the addition of this fixed effect and one random coefficient has significantly improved the fit of the more elaborate model to the data.

The parameter estimates of the observed variables can be interpreted much the same way as those from the standard logistic regression model. For instance, everything else being equal except slight differences on the random effect in the model, under-five children born to mothers' with higher education were $58.3 \%$ less likely to die $(\mathrm{OR}=0.417)$ compared to under-five children born to mothers' with no education controlling for other variables in the model and random effect at level two.

\subsubsection{Multilevel Logistic Regression Model Comparison}

The deviance, likelihood ratio test, AIC and BIC values were used for selecting the best fitting model among the three fitted two level logistic regression models considered. Table 9 shows that the deviance of the empty model with random intercept (4800.7) is greater than the deviance of the random intercept with fixed coefficient (4037.58) and also the deviance of the random intercept with fixed coefficient (4037.58) is greater than the deviance of the random coefficient model (3994.78). These indicate that the random intercept with random coefficient model is better than the multilevel empty model and also the random coefficient model is better than the random intercept with fixed coefficient model.

Similarly, the values of the Akaike Information Criterion (AIC) were used to make an overall comparison of the three models. Table 9 also shows that the AIC value for the random coefficient model is less than that of the random intercept with fixed coefficients model and the empty model with random intercept. This indicates that the random coefficient model provides a better fit as compared to the empty model with 
random intercept and the random intercept with fixed effect model.

Table 9. Results of multilevel logistic regression model selection criteria.

\begin{tabular}{|c|c|c|c|c|c|c|c|}
\hline Model selection criteria & $\begin{array}{l}\text { Log likelihood } \\
\text { (L L) }\end{array}$ & Deviance-2LL & $\begin{array}{l}\text { Deviance based } \\
\text { on chi-square }\end{array}$ & p-value & Df. & AIC & BIC \\
\hline Multilevel Empty model & -2400.35 & 4800.7 & 10.56 & 0.0006 & 2 & 4804.7 & 4819.25 \\
\hline Multilevel Random intercept model & -2018.791 & 4037.58 & 18.9 & 0.000 & 24 & 4085.58 & 4260.12 \\
\hline Multilevel Random coefficient model & -1997.39 & 3994.78 & 42.8 & 0.000 & 26 & 4046.77 & 4235.86 \\
\hline
\end{tabular}

Goodness of fit test

An overall evaluation of the multilevel logistic regression model was assessed using deviance. The test is done by comparing the deviance ( -2 log likelihood) of two models by subtracting the smaller deviance (model with smaller parameters) from the larger deviance (model with greater parameters). The difference is a chi-square test with degrees of freedom equal to the difference in the number of parameters of the two models. The chi-square test results indicate that the model is a good fit. Similarly, the overall model evaluation was assessed using AIC and BIC. Based on the results in Table 9, the Random coefficient model is a good fit.

The likelihood ratio test of no significant difference between the random intercept with fixed effect and random coefficient model gives $\mathrm{LR}=42.8$ (which is the difference between the deviance of the random intercept with fixed effect (4037.58) and the random coefficient (3994.78) with $p=0.000$ This implies that there is a significant difference between the two nested models. Similarly, the values of fit statistics for the random coefficient logistic regression model ( $\mathrm{AIC}=4046.77$ and $\mathrm{BIC}=4235.86$ ) are less than those for the random intercept with fixed coefficient model $(\mathrm{AIC}=4085.583$ and $\mathrm{BIC}=$ 4260.122). These indicate that the random coefficient logistic regression model provides a better fit to the data than the random intercept with fixed effect model. Thus, all model comparison criteria revealed that the random coefficient model is the best fitting model among the three two-level models considered.

\section{Discussion, Conclusion and Recommendations}

In this chapter, we discuss the findings of this study and also draw conclusions and forward recommendations based on the results obtained.

\subsection{Discussion}

This study has attempted to identify the significant socio-economic and demographic factors influencing under-five child mortality and evaluate the variation of these factors among the regional states of Ethiopia using the Ethiopian Demographic and Health Survey (EDHS, 2016) data set. Descriptive statistics, Single-level logistic regression and multilevel logistic regression statistical methods of data analyses were employed to identify determinants of under-five mortality in Ethiopia. The obtained results are discussed as follows.

The results of this study showed that children in Afar and Benishangul-Gumuz regionswere at a higher risk of death than children in Tigray. This study showed that region was a significant factor and some variation of U5CM was observed between regions. Under-five children in Afar, Harari and Dire Dawa were more likely to die compared to under-five children in Tigray region. This disparity could be due to differences in the distribution of socio economic, infrastructure and functioning of the healthcare system in each region. This result is consistent with the finding of [4] in Ethiopia.

The results of this study showed that Female children had reduced risks of dying before 5 years of age compared to male children. Female children were $25 \%$ less likely to experience under-five deaths compared with male children. This finding is complementary to the findings of [4] and [26]. They found out that the risk of death of under- five female children was less than the risk of death of male under-five children.

The results of the present study indicate that age of a child is one of the important determinant factors of under-five child mortality in Ethiopia. For a one month increase in age, the odds of having mortality increased by $1.3 \%(\mathrm{OR}=1.013)$.

The results of this study have revealed that births type has a statistically significant effect on U5CM. Multiple births were more associated with under-five child mortality than single births. This result is consistent with the results of the study by [4]. Similarly, breastfeeding status showed a statistically significant effect on U5CM. Never breastfed under-five childrenuntil the survey time were more likely to die than ever breasted under-five children. This result is consistent with the results of the study by [4] and the study by [29] in Ethiopia. They showed that there is a higher under-five child death among children who were not breastfed than those ever breastfed fed.

Birth order of a child showed a statistically significant effect on under-five child mortality. Some studies showed that first births were at a higher risk of under-five child mortality while others showed that higher ranked births were at increased risk of under-five child mortality. For instance, a study conducted in Taiwan [34] showed that children with first and fifth ranked births were at higher risk of early under-five child mortality. [17] Showed that children with sixth or higher order birth were at increased risk of under-five child mortality in Ethiopia. Our study showed that five or more ranked births were at an increased risk of under-five child mortality compared to first ranked births.

Education plays a significant role in reducing under-five child mortality, particularly maternal education has a greater 
contribution towards the reduction of under-five child mortality. In line with this, [27] found that child mortality in Ethiopia is highly associated with mother's education. The results showed that children born to mothers' with no education experience higher risk of mortality than children born to mothers with primary and higher education. Similarly, [22] in Ethiopia found similar results that mothers' education and under-five child mortality are significantly associated. In this study, it was found that children born to mothers with higher education were less exposed to mortality than children born to mothers with no education.

Household size plays an important role in under-five child mortality. The results of this study revealed that household size has a significant effect on U5CM: Children in households of size 4 and above were less likely to die before the age of 5 compared to children in households of size two or less (below three). This result is consistent with the results of the study by [10] that was carried out in Ethiopia. His results indicated an inverse relationship between under-five child mortality and numbers of household members and contrarily, the study by [29] carried out in Ethiopia found direct relationship between under-five child mortality and number of household members. These results are contrary to our expectation. This discrepancy could be due to the time gap between the current study and the study by [10] and [29].

Place of residence is significantly associated with under-five child mortality. Children born in rural areas were more likely to die than those born in urban areas. This result is consistent with the findings of [27] that children living in rural areas face higher risk of mortality than children living in urban areas. This result also supports the earlier findings of [7] which showed that the risk of under-five mortality for children born in rural areas is almost 66 percent higher than the risk for children born in urban areas.

Finally, this study found out that type of toilet facilities and U5CM are associated. The odds of under-five child mortality among children in households using unimproved toilet facility were more than the odds of under-five child mortality among children in households having improved toilet facilities and children in households with no toilet facilities were much more likely to die compared to under-five children in households with improved toilet facilities, controlling for the other variables in the model. In line with this, [7] found that the risk of under-five mortality is higher for children living in household without any toilet compared to the children living in houses with improved toilet facilities.

\subsection{Conclusion}

This study was intended to identify some determinants of under-five child mortality in Ethiopia based on Ethiopia Demographic and Health Survey (EDHS 2016) data. Accordingly, descriptive analysis, single level logistic regression and multi-level logistic regression analyses techniques were used.

The study included the most important predictor variables that were categorized under socio-economic, demographic and environmental characteristics. The results of this study revealed that Sex of a child, Age of child in months, Birth type, Birth order number, Household size, breastfeeding status, Mothers level of education, Region, Place of residence and type of toilet facility were among the determinants of under-five child mortality in Ethiopia.

The random coefficient model was found to be the best fitting model among the three two-level models considered. Under-five child mortality variation among regional states is accounted by the random intercept of the model. Moreover, the variance of the random component, related to the intercept term, is found to be significant implying the presence of under-five child mortality variation across regional states.

The variation between regions is very small as shown by the findings of this study. This shows that regions tend to perform at comparable levels towards reducing under-five child mortality. However, there is a variation across regional state of Ethiopia up until now.

According to the results of the multilevel logistic regression analysis, the effects of breastfeeding varied across regions whereas the effects of the other covariates on under-five child mortality remained fixed across regions. Thus, we conclude that breastfeeding had significant impacts on under-five child mortality and varies across regional states of Ethiopia. The analysis of the final model indicated significant regional-level variation. This may suggest differences in lifestyles, culture, or environment between different regions. Because of these potential cultural, socio-economic and environmental differences, under-five child mortality exhibits a significant variation among regions of Ethiopia.

\subsection{Recommendations}

Based on the results of the study, the following recommendations can be made.

To reduce more under-five deaths and enhance child survival, efforts by the government and other stakeholders in the health sector must be directed at factors identified by the study.

The results of our study revealed that multiple births are associated with under-five child mortality. This calls for the improvement of maternal and child health services that contribute to improvement of child survival rates.

The severity of U5CM varies from one region to another. Future studies should focus on identifying the risk factors of U5CM for each region of Ethiopia separately in order to inform policy makers to formulate region specific strategy. The government should give more attention to those regions with high under-five mortality rates (like Affar and Benishangul-Gumuz) so that the rate in these regions is substantially reduced.

Breastfeeding plays an important role in child survival. Government and other concerned bodies should give more attention to creating more awareness about the benefits of breastfeeding.

Finally, we recommended that further research should be conducted to identify the determinants of under-five child mortality in Ethiopia including other factors that were not included in this study due to absence of recorded data. 


\section{References}

[1] Agresti, A. (1996). An Introduction to Categorical data Analysis: John Wiley and Sons, Inc., New York.

[2] Agresti, A. (2000). An Introduction to Categorical data Analysis. (2nd Ed.). John Wiley and Sons, Inc. New York.

[3] Agresti, A. (2002). An Introduction to Categorical data Analysis. (2nd Ed.). John Wiley and Sons, Inc. New York.

[4] Bedada, D. (2017). Determinant of Under-Five Child Mortality in Ethiopia. American Journal of Statistics and Probability, 2 (2), 12-18.

[5] Bereka, S. G., \& Habtewold, F. G. (2017). Under-Five Mortality of Children and its Determinants in Ethiopian Somali Regional State, Eastern Ethiopia. Health Science Journal, 11 (3).

[6] Central Statistical Agency (CSA. 2016) [Ethiopia] and ICF International. 2016. Ethiopia Demographic and HealthSurvey 2016. Addis Ababa, Ethiopia and Rockville, Calverton, Maryland, USA: Central Statistical Agency and ICF International.

[7] Chowdhury, A. H. (2013). Determinants of under-five mortality in Bangladesh. Open Journal of Statistics, 3 (03), 213.

[8] Dayton, C. M. (1992). Logistic Regression Analysis; Department of Measurement. Statistics and Evaluation, University of Maryland.

[9] DeLong, E. R., Peterson, E. D., DeLong, D. M., Muhlbaier, L. H., Hackett, S., \& Mark, D. B. (1997). Comparing risk adjustment methods for provider profiling. Statistics in medicine, 16 (23), 2645-2664.

[10] Desta, M. (2011). Infant and Child Mortality in Ethiopia. The role of Socioeconomic, Demographic and Biological factors in the previous five years period of 2000 and 2005.

[11] Ethiopia Demographic and Health Survey (EDHS). Central Statistics Authority \& ORC Marco. Addis Ababa, Ethiopia and Calverton, Maryland, USA, 2016.

[12] Gelman, A, \&Hill, J (2007). Data Analysis Using Regression and Multilevel/Hierarchical Models. Cambridge University Press.

[13] Guo, G., \& Zhao, H. (2000). Multilevel modeling for binary data. Annual review of sociology, 26 (1), 441-462.

[14] Goldstein, H. (1991). Nonlinear multilevel models, with an application to discrete response data. Biometrika, 45-51.

[15] Goldstein, H., \& Rasbash, J. (1996). Improved approximations for multilevel models with binary responses. Journal of the Royal Statistical Society. Series A (Statistics in Society), 505-513.

[16] Goldstein, H. (2003). Multilevel Statistical Models. (3rd Ed.). Oxford University Press. London: Arnold; New York: Oxford University Press Inc.

[17] Goro, M. (2007). The stalling child mortality: the case of three northern regions. In The 5th conference of union for Africa population, Tanzania.

[18] Hailemariam, A., \& Tesfaye, M. (1997). Determinants of infant and early childhood mortality in a small urban community of Ethiopia: a hazard model analysis. Ethiopian Journal of Health Development, 11 (3), 189-200.
[19] Hosmer, D. W., \& Lemeshow, S. (1989). Applied Logistic Regression. John Wiley \& Sons, New York Google Scholar.

[20] Hosmer, D. and Lemeshow, S. (2000). Applied Logistic Regression. (2nd Ed.). John Wiley \& Sons, Inc., New York.

[21] Khan, H. R., \& Shaw, E. (2011). Multilevel logistic regression analysis applied to binary contraceptive prevalence data.

[22] Kumar, P. P., \&File, G. (2010). Infant and child mortality in Ethiopia: a statistical analysis approach. Ethiopian Journal of Education and Sciences, 5 (2).

[23] Lemani, C. (2013). Modelling covariates of infant and child mortality in Malawi (Doctoral dissertation, University of Cape Town).

[24] McCullough, P. and Nelder, J. A. (1989). Generalized Linear Models. Monographs on Statistics and Applied Probability 37. (2nd Ed.). Chapman and Hall, London. UK.

[25] Mosley, W. H., \& Chen, L. C. (1984). An analytical framework for the study of child survival in developing countries. Population and Development Review, 10 (0), 25-45.

[26] Mugarura, A., \& Kaberuka, W. (2015). Multilevel analysis of factors associated with child mortality in Uganda. African Journal of Economic Review, 3 (2), 125-139.

[27] Senayit, S., \& Eshetu, W. (2013). Determinants of mortality among one to four years old children in Ethiopia: A study based on the 2011 EDHS data. Ethiopian Journal of Health Development, 27 (1), 8-15.

[28] Seyoum, Y., \& Sharma, M. K. (2014). Survival Analysis of Under Five Mortality in Rural Parts of Ethiopia. International Journal of Statistics in Medical Research, 3 (3), 266-281.

[29] Solomon, G., \& Emmanuel, G. (2016). Determinants of Under-Five Mortality in High Mortality Regions of Ethiopia: An Analysis of the 2011 Ethiopia Demographic and Health Survey Data. International Journal of Population Research, 2016.

[30] Twum-Baah (1994) A study of infant, child and maternal mortality in Ghana. Ghana statistical services in collaboration with Ministry of Health and UNICEF, Accra, Ghana 24-32.

[31] UNICEF. (2012). Levels and trends in child mortality. Estimates developed by the UN Inter-agency Group for Child Mortality Estimation (IGME).

[32] UNICEF. (2017). Levels and trends in child mortality. Estimates developed by the UN Inter-agency Group for Child Mortality Estimation (IGME).

[33] Van Raalte, A. A., Kunst, A. E., Lundberg, O., Leinsalu, M., Matikainen, P., Artnik, B., Deboosere, P., Stirbu, I., Wojtyniak, B. and Mackenbach, J. P. (2012). The contribution of educational inequalities to lifespan variation. Population Health Metrics, 2012, 10 (3): 1-12.

[34] Wang PD, Lin RS. Perinatal mortality in Taiwan. Public Health 1999; 113-33.

[35] World Health Organization, \& UNICEF. (2012). Trends in maternal mortality: 1990 to 2010: WHO, UNICEF, UNFPA and the World Bank estimates.

[36] Yifang, H (2013). The analysis of child mortality in a developing country. Stockholm University. Unpublished master's thesis. 